A. Sannami

Nagoya Math. J.

Vol. 90 (1983), 1-55

\title{
THE STABILITY THEOREMS FOR DISCRETE DYNAMICAL SYSTEMS ON TWO-DIMENSIONAL MANIFOLDS
}

\author{
ATSURO SANNAMI
}

\section{§1. Introduction}

One of the basic problems in the theory of dynamical systems is the characterization of stable systems.

Let $M$ be a closed (i.e. compact without boundary) connected smooth manifold with a smooth Riemannian metric and $\operatorname{Diff}^{r}(M)(r \geq 1)$ denote the space of $C^{r}$ diffeomorphisms on $M$ with the uniform $C^{r}$ topology. Let $f \in \operatorname{Diff}^{s}(M)$ with $s \geq r$. Then $f$ is called $C^{r}$ structurally stable if and only if there is a neighborhood $\mathscr{U}(f)$ of $f$ in $\operatorname{Diff}^{r}(M)$ such that for any $g \in \mathscr{U}(f)$ there exists a homeomorphism $h: M \rightarrow M$ satisfying $g h=h f$.

Another important notion of stability is the $\Omega$-stability. Recall that $x \in M$ is a non-wandering point of $f$ if and only if for any neighborhood $U$ of $x$, there is a nonzero integer $m$ such that $f^{m}(U) \cap U \neq \phi$. The set $\Omega(f)$ of all the non-wandering points of $f$ is a closed invariant set. $f$ is called $C^{r} \Omega$-stable if and only if there is a neighborhood $\mathscr{U}(f)$ of $f$ in $\operatorname{Diff}^{r}(M)$ such that for any $g \in \mathscr{U}(f)$ there exists a homeomorphism $h: \Omega(f)$ $\rightarrow \Omega(g)$ satisfying $g h=h f$ on $\Omega(f)$.

The essential condition to characterize these stabilities is "Axiom $A$ " introduced by S. Smale in [17]. Namely, $f$ satisfies Axiom $A$ if and only if

(a) $\Omega(f)$ is a hyperbolic set,

(b) $\overline{\operatorname{Per}(f)}=\Omega(f)$,

where $\operatorname{Per}(f)$ denotes the set of all the periodic points of $f$. Recall that a compact $f$-invariant subset $\Lambda \subset M$ is a hyperbolic set if and only if there exist constants $c>0,0<\lambda<1$ and a $T$ f-invariant splitting $T M \mid \Lambda=E^{s}$ $\oplus E^{u}$ such that

$$
\begin{aligned}
& \left\|T f^{n} \mid E_{p}^{s}\right\| \leq c \lambda^{n} \\
& \left\|T f^{-n} \mid E_{p}^{u}\right\| \leq c \lambda^{n}
\end{aligned}
$$

for all $p \in \Lambda$ and non-negative integers $n$.

\footnotetext{
Received April 22, 1981.
} 
In [9], [18] and [8], the following is conjectured.

Structural stability conjecture. $f$ is $c^{r}$ structurally stable if and only if $f$ satisfies Axiom $A$ and Strong transversality condition.

$\Omega$-stability conjecture. $f$ is $c^{r} \Omega$-stable if and only if $f$ satisfies Axiom $A$ and No cycle property.

For the definitions of Strong transversality condition and No cycle property, we refer to [9], [18].

The purpose of this paper is to give an affirmative answer to these conjectures for $f$ of class $C^{2}$ in case of $\operatorname{dim} M=2$ and $r=1$.

The sufficiency parts are known to be true for arbitrary dimension, that is; if $f$ satisfies Axiom $A$ and Strong transversality condition, then $f$ is $C^{1}$ structurally stable (Robbin [13] for $f$ of class $C^{2}$ and Robinson [15] for $f$ of class $C^{1}$ ), and if $f$ satisfies Axiom $A$ and No cycle property, then $f$ is $C^{1} \Omega$-stable (Smale [18]). Remark that if $f$ is $C^{1}$ structurally (resp. $\Omega$-) stable and of class $C^{s}$, then $C^{r}$ structurally (resp. $\Omega$-) stable for all $1 \leq r \leq s$.

Concerning the converses, it is known that $C^{r}$ structural stability plus Axiom $A$ implies Strong transversality condition [14], and $C^{r} \Omega$ stability plus Axiom $A$ implies No cycle property [8], for all $r \geq 1$. Thus, if we can verify that $\Omega$-stability implies Axiom $A$, then the above conjectures are established.

In this paper, we investigate a certain class $F(M)$ of $C^{1}$ diffeomorphisms introduced by Mañé [5], [6] which contains all $C^{1} \Omega$-stable diffeomorphisms; namely, we put

$F(M)=\operatorname{int}_{1}\left\{g \in \operatorname{Diff}^{1}(M)\right.$ : any periodic point of $g$ is hyperbolic $\}$, where int ${ }_{1}$ means "interior" with respect to $C^{1}$ topology of $\operatorname{Diff}^{1}(M)$ and a periodic point $x$ of $g$ with period $m$ is hyperbolic if and only if $T_{x} g^{m}: T_{x} M \rightarrow T_{x} M$ has no eigenvalue of absolute value one.

Our result is the following;

Theorem. Let $\operatorname{dim} M=2$ and $f \in F(M)$. If $f$ is of class $C^{2}$, then $\Omega(f)$ is a hyperbolic set.

By the theorem of Kupka-Smale [14], it can be seen that if $f$ is $C^{1}$ $\Omega$-stable, then $f \in F(M)$. Furthermore, by the " $C$ 1 Closing lemma", we have $\overline{\operatorname{Per}(f)}=\Omega(f)$, namely Axiom $A(b)$ holds for $f \in F(M)$, (see Lemma 
3.1 in [6] for the proof).

Thus, as corollaries of our Theorem, we get;

Structural stability theorem. Let $\operatorname{dim} M=2$ and $f \in \operatorname{Diff}^{2}(M) . \quad f$ is $C^{1}$ structurally stable if and only if $f$ satisfies Axiom $A$ and strong transversality condition.

$\Omega$-stability theorem. Let $\operatorname{dim} M=2$ and $f \in \operatorname{Diff}^{2}(M) . \quad f$ is $C^{1} \Omega$-stable if and only if $f$ satisfies Axiom $A$ and No cycle property.

In this paper, we investigate only $C^{1}$ stability, namely $F(M)$, because the " $C^{r}$ Closing lemma" has not been established for $r \geq 2$. As we mentioned above, for the proof of Axiom $A(b)$ for $f \in F(M)$, we need the " $C^{1}$ Closing lemma", and this is again our main tool for the proof of our Theorem. As is pointed out by Robinson in [14], it seems to be an interesting problem whether $C^{2}$ structural (or $\Omega$-) stability implies $C^{1}$ structural (or $\Omega$-) stability or not.

This paper consists of 15 sections. In Sections $2 \sim 9$, we give technical preliminaries. The essential part of the proof of our Theorem is given in Sections $10 \sim 15$.

For the understanding of the basic ideas of the proof, the reader is recommended to look at the statements of (2.3), (4.1), (12.1), (12.2), (12.3) and all of Section 13; "Proof of the Theorem". Then it will be understood how easily the Theorem may be proved provided that (12.2) Lemma and (12.3) Lemma have been verified. The essence is in (12.2) Lemma and it is proved without much difficulty, once (11.1) Key lemma is established. Therefore, we will expend our almost all efforts in proving this Key lemma.

The present work is motivated by Mañe [5], Robinson [16] and Pliss [10], [11], especially, Pliss's excellent idea of "cutting off the suborbits" in [10]. We apply it to the proof of our (11.2) Main lemma in Section 15, but in his argument, there are some points which are not clear and we use this idea in a different way from his.

After I had finished this work, I was informed that in [Chin. Ann. of Math. 1(1980), 9-29.] S. D. Liao also asserted that he proved the stability conjectures for $C^{1}$ diffeomorphisms on 2-manifolds and for $C^{1}$ flows with isolated singularities on 3-manifolds. But his method is considerably different from ours.

I would like to thank Haruo Suzuki, Kenichi Shiraiwa and Takashi Sakai for helpful advices and supports. Especially, Masahiro Kurata 
must be thanked for his guidance and many valuable suggestions.

\section{LIST OF Symbols}

$\begin{array}{ll}\text { [ ]: integer part of a number. } & r_{1}:(5.4) . \\ d(x, y): \text { distance from } x \text { to } y . & r_{2}:(5.4) . \\ Z_{+}: \text {non-negative integers. } & r_{3}:(6.1) . \\ a_{p}: \S 5 . & r_{4}:(6.2) . \\ A_{0}:(11.1) . & r_{5}: \S 7 . \\ b_{p}\left(v, a_{1}, a_{2}\right): \S 7 . & r_{6}:(9.1) . \\ B(r), B\left(r^{\prime}, r^{\prime \prime}\right): \S 6 . & r_{7}:(9.1) . \\ B_{p}(r), B_{p}\left(r^{\prime}, r^{\prime \prime}\right): \S 6 . & r_{8}:(10.2) . \\ c:(2.3),(2.4),(2.5) . & r_{9}:(14.4) . \\ c_{1}:(5.4)(\mathrm{vi}) . & T\left(r, r^{\prime}\right):(14.1) . \\ d_{p}: \S 5 . & T_{p}\left(r, r^{\prime}\right):(14.3) . \\ E_{p}^{u}(f), E_{p}^{s}(f): \S 2 . & U(r):(5.4)(\mathrm{i}) . \\ E^{1}, E^{2}, E_{p}^{1}, E_{p}^{2}:(2.3)(\mathrm{ii}) . & \mathscr{U}_{1}:(2.3)(\mathrm{i}) . \\ F(M): \S 1 . & \mathscr{U}_{2}:(2.9) . \\ G:(10.1) . & \mathscr{U}_{(\varepsilon, f): \S 2 .} . \\ G_{1}:(11.2) . & V_{p}(r): \S 5 . \\ m_{0}:(2.7), \S 5 . & \beta=\lambda^{-1 / 2}: \S 14 . \\ m_{1}: \text { proof of }(10.3) . & \varepsilon_{0}:(10.1) . \\ M_{0}:(5.4)(v) . & \eta(p, n): \S 6 . \\ M_{1}: \S 5 . & \theta(p, n): \S 6 . \\ N_{*}:(11.2) . & \lambda:(2.3),(2.4),(2.5) . \\ N_{0}:(10.1) . & \Lambda=\Lambda_{1}(f): \S 4 . \\ N_{1}:(9.1) . & \Lambda_{i}(f):(2.1) . \\ N_{2}:(10.1) . & \nu_{0}(N):(11.2) . \\ N_{3}:(10.2) . & \omega(p, n): \S 6 . \\ N_{4}:(12.2) . & \end{array}$

\section{§2. Some preliminary results}

From now on, we assume that $M$ is a fixed closed 2-dimensional smooth manifold with a smooth Riemannian metric. For $f \in F(M), p \in$ $\operatorname{Per}(f)$ is hyperbolic and we denote by $E_{p}^{u}(f)\left(E_{p}^{s}(f)\right)$ the unstable (stable) subspace of $T_{p}(M)$. We put

$$
\Lambda_{i}(f)=\text { closure }\left\{p \in \operatorname{Per}(f): \operatorname{dim} E_{p}^{s}(f)=i\right\} \quad \text { for } i=0,1,2 .
$$


Note that $\Lambda_{0}(f)$ and $\Lambda_{2}(f)$ are the sets of all the sources and the sinks of $f$ respectively. In [11], Pliss proved the following,

(2.2) Lemma. For $f \in F(M), \Lambda_{0}(f)$ and $\Lambda_{2}(f)$ are finite sets.

From this lemma, we know that $\Lambda_{0}(f)$ and $\Lambda_{2}(f)$ are hyperbolic sets. The following lemma proved by Mañe [5] will play a key role in studying the precise properties of $f \in F(M)$.

(2.3) Lemma. For $f \in F(M)$, there exist $c>0$ and $0<\lambda<1$ satisfying;

(i) there exists a $C^{1}$-neighborhood $\mathscr{U}_{1}$ of $f$ such that

$$
\begin{aligned}
& \left\|T g^{\pi(p)} \mid E_{p}^{s}(g)\right\| \leqq c \lambda^{\pi(p)} \\
& \left\|T g^{-\pi(p)} \mid E_{p}^{u}(g)\right\| \leqq c \lambda^{\pi(p)}
\end{aligned}
$$

for all $g \in \mathscr{U}_{1}$ and $p \in \operatorname{Per}(g)$, where $\pi(p)$ denotes the period of $p$.

(ii) there exists a Tf-invariant continuous splitting $T M \mid \Lambda_{1}(f)=E^{1} \oplus E^{2}$ such that

$$
\left\|T f^{n}\left|E_{p}^{2}\|\cdot\| T f^{-n}\right| E_{f^{n}(p)}^{1}\right\| \leqq c \lambda^{n}
$$

for all $n \in Z_{+}$and $p \in \Lambda_{1}(f)$. Moreover if $p \in \Lambda_{1}(f) \cap \operatorname{Per}(f)$, then $E_{p}^{1}=E_{p}^{u}(f)$ and $E_{p}^{2}=E_{p}^{s}(f)$, where $E_{p}^{i}(i=1,2)$ denotes the fiber of $E^{i}$ over $p$.

Note that $\operatorname{dim} E_{p}^{1}=\operatorname{dim} E_{p}^{2}=1$ for all $p \in \Lambda_{1}(f)$.

By changing $0<\lambda<1$ if necessary, we may assume that the constant $c>0$ of (2.3) (i) equals 1 on saddles, that is;

(2.4) LeMma.

$$
\begin{aligned}
& \left\|T g^{\pi(p)} \mid E_{p}^{s}(g)\right\| \leqq \lambda^{\pi(p)} \\
& \left\|T g^{-\pi(p)} \mid E_{p}^{u}(g)\right\| \leqq \lambda^{\pi(p)}
\end{aligned}
$$

for all $g \in \mathscr{U}_{1}$ and $p \in \Lambda_{1}(f) \cap \operatorname{Per}(f)$.

To simplify the notations and calculations in the succeeding sections, we also assume that,

$$
\begin{aligned}
& \text { (i) } 1 / 2 \leqq \lambda<1 \quad \text { and } \\
& \text { (ii) } c \geqq 1
\end{aligned}
$$

(i) will be used only to formulate (14.5). Throughout this paper, the symbols $c$ and $\lambda$ denote the constants given by (2.3), (2.4), and (2.5).

The next lemma is a modification of Lemma 2.1 in [10]. 
(2.6) Lemma. Let $n$ be a positive integer and $h:\{0,1,2, \cdots, n\} \rightarrow \boldsymbol{R}$ be a map. Take $m \leqq \min _{0 \leq i \leq n-1}\{h(i+1)-h(i)\}$, and $\sigma>m$. If $h(n)-$ $h(0) \leqq \ell$ for some $\ell \in R$ with $n \sigma-\ell \geqq 0$, then there exist $\nu=[(n \sigma-\ell) \mid$ $(\sigma-m)]+1$ integers $0 \leq k_{1} \leq k_{2}<\cdots<k_{\nu} \leq n$ such that

$$
h\left(k+k_{j}\right)-h\left(k_{j}\right) \leq k \sigma
$$

for all $0 \leq k \leq n-k_{j}$ and $1 \leq j \leq \nu$.

Proof. We shall select $k_{f}$ inductively as follows. Let $k_{1}$ be the largest integer in $\{0,1, \cdots, n\}$ satisfying $h\left(k_{1}\right)-h(0) \geq k_{1} \sigma$. Such an integer exists, because zero satisfies this condition. $k_{1}$ has the property that

$$
h\left(k+k_{1}\right)-h\left(k_{1}\right) \leq k \sigma \quad \text { for all } 0 \leq k \leq n-k_{1},
$$

because if $h\left(k+k_{1}\right)-h\left(k_{1}\right)>k \sigma$ for some $0<k \leq n-k_{1}$, then

$$
h\left(k+k_{1}\right)-h(0)=h\left(k+k_{1}\right)-h\left(k_{1}\right)+h\left(k_{1}\right)-h(0)>\left(k_{1}+k\right) \sigma
$$

which contradicts the definition of $k_{1}$.

Assume that $k_{j}$ is defined and $k_{j}<n$. We select $k_{j+1}$ as the largest integer in $\left\{k_{j}+1, k_{j}+2, \cdots, n\right\}$ satisfying

$$
h\left(k_{j+1}\right)-h\left(k_{j}+1\right) \geq\left(k_{j+1}-k_{j}-1\right) \sigma .
$$

Such an integer exists because $k_{j}+1$ satisfies this condition. By the same argument as above, we have,

$$
h\left(k+k_{j+1}\right)-h\left(k_{j+1}\right) \leq k \sigma \quad \text { for all } 0 \leq k \leq n-k_{j+1} .
$$

Now we shall estimate how many $\left\{k_{j}\right\}$ we can select. Since

$$
\begin{aligned}
\ell \geq & h(n)-h(0)=\left(h(n)-h\left(k_{j}\right)\right)+\left(h\left(k_{j}\right)-h\left(k_{j-1}+1\right)\right) \\
& +\left(h\left(k_{j-1}+1\right)-h\left(k_{j-1}\right)\right)+\cdots+\left(h\left(k_{1}+1\right)-h\left(k_{1}\right)\right) \\
& +\left(h\left(k_{1}\right)-h(0)\right) \\
\geq & \left(n-k_{j}\right) m+\left(k_{j}-k_{j-1}-1\right) \sigma+m+\cdots+m+k_{1} \sigma \\
= & n m-(j-1)(\sigma-m)+k_{j}(\sigma-m),
\end{aligned}
$$

and $\sigma-m>0$, we have

$$
k_{j} \leq(j-1)+(\ell-n m) /(\sigma-m) .
$$

Namely, if $j \leq(n \sigma-\ell) /(\sigma-m)$, we can define $k_{j+1}$ because

$$
\begin{aligned}
k_{j} & \leq(n \sigma-\ell) /(\sigma-m)-1+(\ell-n m) /(\sigma-m) \\
& =n-1 .
\end{aligned}
$$


This completes the proof.

Applying (2.6) to our situation with $h(k)=\log \left\|T f^{k} \mid E_{p}^{1}\right\|$, we get the following,

(2.7) Lemma. Let $f \in F(M), p \in \Lambda_{1}(f)$ and $n$ be a positive integer. Put $m_{0}=\inf _{p \in A_{1}(f)}\left\{\left\|T f\left|E_{p}^{1}\|\| T f,\right| E_{p}^{2}\right\|\right\}$, and take $\rho>m_{0}$. If $\log \left\|T f^{n} \mid E_{p}^{1}\right\| \leq \ell$ for some $\ell \in R$ with $n(\log \rho) \geq \ell$, then there are

$$
\nu=\left[(n(\log \rho)-\ell) /\left(\log \rho-\log m_{0}\right)\right]+1
$$

integers $0 \leq k_{1}<k_{2}<\cdots<k_{\nu} \leq n$ such that

$$
\left\|T f^{k} \mid E_{f^{k_{j}(p)}}^{1}\right\| \leq \rho^{k}
$$

for all $0 \leq k \leq n-k_{j}$ and $1 \leq j \leq \nu$.

Now we shall give here a $C^{1}$-distance on a neighborhood of $f \in$ $\operatorname{Diff}^{1}(M)$. The definition is a usual one.

Let $\left\{U_{i}, \alpha_{i}\right\}_{i \in I}$ and $\left\{V_{j}, \beta_{j}\right\}_{j \in J}$ be local coordinate systems on $M$ with finite index sets, and $\gamma: I \rightarrow J$ be a map such that $f\left(\bar{U}_{i}\right) \subset V_{\gamma^{(i)}}$ for all $i \in I$. Let $g_{1}, g_{2} \in \operatorname{Diff}^{1}(M)$ be sufficiently near $f$ so that $g_{k}\left(\bar{U}_{i}\right) \subset V_{r(i)}$ for all $i \in I(k=1,2)$. We define a $C^{1}$-distance on a neighborhood of $f$ by

$$
\begin{aligned}
d_{1}\left(g_{1}, g_{2}\right)=\sup _{i \in I} \sup _{x \in \alpha_{i}\left(U_{i}\right)} \max \left\{\left|\beta_{\gamma(i)} g_{1} \alpha_{i}^{-1}(x)-\beta_{\gamma^{(i)}} g_{2} \alpha_{i}^{-1}(x)\right|,\right. \\
\left.\left\|T_{x}\left(\beta_{\gamma^{(i)}} g_{1} \alpha_{i}^{-1}\right)-T_{x}\left(\beta_{\gamma^{(i)}} g_{2} \alpha_{i}^{-1}\right)\right\|\right\} .
\end{aligned}
$$

With this distance we can define an $\varepsilon$-neighborhood of $f$ for small $\varepsilon>0$, which is denoted by $\mathscr{U}(\varepsilon, f)$.

Clearly we have the next lemma which guarantees simultaneous perturbations with disjoint supports, that is;

(2.8) Lemma. Let $\varepsilon>0$ be small and $g_{1}, g_{2} \in \mathscr{U}(\varepsilon, f)$. If $\operatorname{supp}\left(g_{1} f^{-1}\right) \cap$ $\operatorname{supp}\left(g_{2} f^{-1}\right)=\phi$, then we have;

$$
\left(g_{1} f^{-1}\right)\left(g_{2} f^{-1}\right) f \in \mathscr{U}(\varepsilon, f) .
$$

Next lemma is an easy modification of (1.1) Lemma in [1], and will be used in Sections 3 and 8 .

(2.9) Lemma. Let $f \in \operatorname{Diff}^{1}(M)$. There exists a $C^{1}$-neighborhood $\mathscr{U}_{2}$ of $f$ satisfying the following: For any $\varepsilon>0$, there exists $\delta>0$ such that, if $\left\{p_{1}, \cdots, p_{m}\right\}$ is a finite set of points in $M, g \in \mathscr{U}_{2}$ and $A_{i}(i=1, \cdots, m)$ are linear automorphisms on $T_{g\left(p_{i}\right)} M$ satisfying $\left\|A_{i}-\mathrm{id}\right\| \leq \delta$ for all $1 \leq i \leq m$, then there exists a $C^{1}$ map $g^{\prime}$ such that 
(i ) $d_{1}\left(g, g^{\prime}\right)<\varepsilon$

(ii) $\left(T g^{\prime}\right)_{p_{i}}=A_{i} \circ(T g)_{p_{i}}$ for all $1 \leq i \leq m$.

Moreover, for any neighborhood $U$ of $\left\{p_{1}, \cdots, p_{m}\right\}$, we can require that $\mathrm{g}=\mathrm{g}^{\prime}$ outside $U$.

\section{§3. Stability of sinks and sources}

Throughout the rest of this paper, $f$ denotes a fixed $C^{2}$ diffeomorphism in $F(M)$. In this section, we shall show that the sinks and sources of $f$ are stable under small perturbations.

(3.1) Lemma. Given $\varepsilon>0$, there is a $C^{1}$-neighborhood $\mathscr{U}$ of $f$ with the following property; for any $g \in \mathscr{U}$, there is a bijection $h: \Lambda_{0}(f) \cup \Lambda_{2}(f) \rightarrow$ $\Lambda_{0}(g) \cup \Lambda_{2}(g)$ such that $d_{0}(h, \mathrm{id}) \leq \varepsilon$, where $d_{0}$ denotes the $C^{0}$ distance.

Remark that if $f$ is structurally stable, this lemma is obvious by the stability of hyperbolic sets [3]. But for $f \in F(M)$, it is not so easy, because we must guarantee that there appear neither new sinks nor sources under small perturbations. We may find an idea of our proof in [11]. Using (2.3) and (2.9), we can modify Theorem 3.1 in [11] as follows.

(3.2) LEMma. There exist a $C^{1}$-neighborhood $\mathscr{U}$ of $f$ and $\theta_{0}>0$ satisfying the following; for any integer $\theta \geq \theta_{0}$, there is $m(\theta) \geq 0$ such that for $g \in \mathscr{U}$ and $p \in \Lambda_{2}(g)$ with $\pi(p) \geq m(\theta)$, we have

$$
\prod_{i=1}^{[\pi(p) / \theta]}\left\|\left(T g^{\theta}\right)_{g^{(i-1) \theta(p)}}\right\| \leq\left(\lambda^{1 / 2}\right)^{\pi(p)}
$$

where $\pi(p)$ denotes the period of $p$.

Proof of (3.1). We only need to prove the lemma for $\Lambda_{2}$, that is; for any $\varepsilon>0$ there is a $C^{1}$-neighborhood $\mathscr{U}$ of $f$ such that for any $g \in \mathscr{U}$, there exists a bijection $h: \Lambda_{2}(f) \rightarrow \Lambda_{2}(g)$ with $d_{0}(h$, id $) \leq \varepsilon$.

Suppose the contrary, i.e., there exist $\varepsilon>0$ and sequences $g_{n} \rightarrow f$, $p_{n} \in \Lambda_{2}\left(g_{n}\right)$ such that $d\left(p_{n}, \Lambda_{2}(f)\right)>\varepsilon$.

If $\pi\left(p_{n}\right)$ are bounded as $n \rightarrow \infty$, by taking a subsequence we can assume that there is a $k \in Z_{+}$with $\pi\left(p_{n}\right)=k$ for all $n$ and $p_{n} \rightarrow p$ for some $p$. Clearly $f^{k}(p)=p$. Since $d\left(p, \Lambda_{2}(f)\right) \geq \varepsilon, p$ is not a sink of $f$. As $p$ is hyperbolic, there is a non-zero vector $v \in T_{p} M$ such that the norm of $T f^{i}(v)$ increase exponentially as $i \rightarrow \infty$. This is a contradiction because $p_{n}$ is a sink of $g_{n}$ and $g_{n} \rightarrow f$. 
Next, we see that the period of $p_{n}$ are bounded as $n \rightarrow \infty$. We can apply the same technique which Pliss used to prove the finiteness of sinks and sources of $f \in F(M)$ in [11]. He showed that the period of sinks of the diffeomorphism in $F(M)$ cannot be arbitrarily large. Using (3.2), we can estimate the supremum of the period of the sinks uniformly in a $C^{1}$-neighborhood of $f$, and get the boundedness of $\left\{\pi\left(p_{n}\right)\right\}$. This completes the proof.

\section{§4. Preliminary lemmas about the spectrum of $f_{*}$}

In what follows, we put $\Lambda=\Lambda_{1}(f)$. Let

$$
\Sigma^{b}\left(E^{i}\right)=\left\{\text { bounded sections } \Lambda \rightarrow E^{i}\right\} \quad i=1,2,
$$

and let $f_{*}: \Sigma^{b}\left(E^{i}\right) \rightarrow \Sigma^{b}\left(E^{i}\right)$ be defined by

$$
f_{*}(\sigma)=T f \circ \sigma \circ f^{-1} \quad \sigma \in \Sigma^{b}\left(E^{i}\right) .
$$

$\Sigma^{b}\left(E^{i}\right)$ is a Banach space with the natural sup. norm and $f_{*}$ is the automorphism of $\Sigma^{b}\left(E^{i}\right)$ canonically induced by $f$.

In this section we shall prove two lemmas that will be used in Sections 5 and 13 .

The next lemma is a modification of an argument in [5].

(4.1) Lemma. Let $\mu>0$. If

$$
\text { spec. } \operatorname{rad} . f_{*}^{-1} \mid \Sigma^{b}\left(E^{1}\right) \geq \mu,
$$

then there is a recurrent point $p_{*} \in \Lambda$ such that

$$
\left\|T f^{n} \mid E_{p_{*}}^{1}\right\| \leq \mu^{-n} \quad \text { for all } n \in Z_{+},
$$

where spec. rad. denotes the spectral radius.

Proof. Let $S$ be the family of compact $f$-invariant subsets of $\Lambda$ such that

$$
\text { spec. rad. } f_{*}^{-1} \mid \Sigma^{b}\left(E^{1} \mid K\right) \geq \mu \quad K \in S .
$$

We define an order on $S$ by inclusion. Then $S$ is an inductively ordered set, and by Zorn's lemma, we may find a minimal element $K_{0} \in S$. By an argument in [7], there is a sequence $\sigma_{n} \in \Sigma^{b}\left(E^{1} \mid K_{0}\right)$ such that $\left\|\sigma_{n}\right\|=1$ and

$$
\left\|\left(f_{*}-r I\right) \sigma_{n}\right\| \leq 1 / n,
$$

where $r=$ (spec. rad. $\left.f_{*}^{-1} \mid \Sigma^{b}\left(E^{1} \mid K_{0}\right)\right)^{-1}$, and $I$ denotes the identity. Note that $r \leq \mu^{-1}$. 
Choose $p_{n} \in \Lambda$ such that $\left|\sigma_{n}\left(p_{n}\right)\right| \geq n /(n+1), p_{n} \rightarrow p_{*}$ for some $p_{*} \in \Lambda$ and $\sigma_{n}\left(p_{n}\right) \rightarrow v \in E_{p_{*}}^{1} \quad$ Clearly $|v|=1$.

Now we shall prove

$$
\left\|T f^{m} \mid E_{p_{*}}^{1}\right\| \leq r^{m} \quad \text { for all } m \in Z_{+} .
$$

Put $L=f_{*} \mid \Sigma^{b}\left(E^{1} \mid K_{0}\right)$ and $w=\max \left\{\sup _{0 \leq k \leq m}\left\|L^{k}\right\|, 1\right\}$, where $m$ is now fixed. Then we have

$$
\begin{aligned}
\left\|\left(L^{m}-r^{m} I\right) \sigma_{n}\right\| & =\left\|\left(L^{m}-r L^{m-1}+r L^{m-1}-r^{m} I\right) \sigma_{n}\right\| \\
& \leq w / n+r\left\|\left(L^{m-1}-r^{m-1} I\right) \sigma_{n}\right\|,
\end{aligned}
$$

and therefore

$$
\begin{aligned}
\left\|\left(L^{m}-r^{m} I\right) \sigma_{n}\right\| & \leq(w / n)\left(1+r+r^{2}+\cdots+r^{m-2}+r^{m-1}\right. \\
& =(w / n) \cdot\left(1-r^{m}\right) /(1-r) .
\end{aligned}
$$

Since $\operatorname{dim} E^{1}=1$ and $|v|=1$, it suffices to show that

$$
\left|T f^{m}(v)\right| \leq r^{m}
$$

In fact,

$$
\begin{aligned}
\left|T f^{m} \sigma_{n}\left(p_{n}\right)\right| & =\left|T f^{m} \sigma_{n} f^{-m}\left(f^{m}\left(p_{n}\right)\right)-r^{m} \sigma_{n}\left(f^{m}\left(p_{n}\right)\right)+r^{m} \sigma_{n}\left(f^{m}\left(p_{n}\right)\right)\right| \\
& \leq\left\|\left(L^{m}-r^{m} I\right) \sigma_{n}\right\|+r^{m} .
\end{aligned}
$$

Since $\sigma_{n}\left(p_{n}\right) \rightarrow v$ and $\left\|\left(L^{m}-r^{m} I\right) \sigma_{n}\right\| \rightarrow 0$ as $n \rightarrow \infty$, we have $\left|T f^{m}(v)\right| \leq r^{m}$ as claimed.

We can also see that $\omega\left(p_{*}\right) \in S$, where $\omega\left(p_{*}\right)$ denotes the $\omega$-limit set of $p_{*}$. Since $K_{0}$ is a minimal element in $S, \omega\left(p_{*}\right)=K_{0}$. This implies that $p_{*}$ is a recurrent point, and completes the proof.

The next lemma is necessary to prove that the pre-laminations $\left\{W_{p}^{1}\right\}_{p \in \Lambda}$ and $\left\{W_{p}^{2}\right\}_{p \in A}$ are of class $C^{2}$ in Section 5 .

(4.2) LEMMA.

$$
\begin{aligned}
& \text { spec. } \operatorname{rad} . f_{*}^{-1} \mid \Sigma^{b}\left(E^{1}\right) \leq 1 \\
& \text { spec. rad. } f_{*} \mid \Sigma^{b}\left(E^{2}\right) \leq 1
\end{aligned}
$$

Proof. We shall only prove the first case.

Suppose that there is a $\mu>1$ such that

$$
\text { spec. } \operatorname{rad} . f_{*}^{-1} \mid \Sigma^{b}\left(E^{1}\right) \geq \mu \text {. }
$$

Then from (4.1), there is a recurrent point $p_{*} \in \Lambda$ such that 


$$
\left\|T f^{n} \mid E_{p_{*}}^{1}\right\| \leq \mu^{-n} \quad \text { for all } n \in Z_{+} .
$$

From (2.3) (ii), we have

$$
\left\|T f^{n} \mid E_{p_{*}}^{2}\right\| \leq c\left(\lambda \mu^{-1}\right)^{n} \quad \text { for all } n \in Z_{+} .
$$

This implies that a neighborhood of $p_{*}$ contracts exponentially under the iteration of $f$. Using the recurrence of $p_{*}$ with precise but straightforward arguments, we can see that there is a sink sufficiently near $p_{*}$. This contradicts (2.2).

\section{§5. Semi-invariant coordinate}

In the succeeding sections, we study the behavior of the iterated images of a neighborhood of a point in 1 . A certain local coordinate system is indispensable for the precise estimates, and we shall define it in this section.

The definition is not a peculiar but a natural one. This local coordinate is defined on a neighborhood of each point $p \in A$, and has the laminae $W_{p}^{1}, W_{p}^{2}$ of the locally $f$-invariant $C^{2}$ prelaminations $\left\{W_{p}^{1}\right\}_{p \in A},\left\{W_{p}^{2}\right\}_{p \in A}$ as the $x$-axis and the $y$-axis of $R^{2}$ respectively. Thus, with respect to this coordinate, $f$ preserves $x$ and $y$-axes.

The arguments in this section heavily depend on Section 5 (especially (5.1) Theorem and (5.5) Theorem) in [4], so refer to it for details.

Recall that, from (2.3) (ii), we have,

$$
\left\|T f^{n}\left|E_{p}^{2}\|\cdot\| T f^{-n}\right| E_{f^{n}(p)}^{1}\right\| \leq c \lambda^{n} \quad \text { for all } n \in Z_{+} \text {and } p \in \Lambda .
$$

Moreover, we have the following,

(5.1) Lemma. There is an $n_{0} \in Z_{+}$such that if $n \geq n_{0}$,

$$
\left\|T f^{n}\left|E_{p}^{2}\|\cdot\| T f^{-n}\right| E_{f^{n}(p)}^{1}\right\|^{k} \leq c\left(\lambda^{1 / 2}\right)^{n}
$$

for all $p \in \Lambda$ and $k=1,2$.

Proof. For $k=1$, this is just (2.3) (ii).

We shall show the case of $k=2$. From (4.2), we have

$$
\begin{aligned}
1 & \geq \text { spec. } \operatorname{rad} . f_{*}^{-1} \mid \Sigma^{b}\left(E^{1}\right) \\
& =\lim _{n \rightarrow \infty}\left\|f_{*}^{-n} \mid \Sigma^{b}\left(E^{1}\right)\right\|^{1 / n} .
\end{aligned}
$$

Hence, there exists $n_{0} \in Z_{+}$such that if $n \geq n_{0}$, 


$$
\left\|f_{*}^{-n} \mid \Sigma^{b}\left(E^{1}\right)\right\|^{1 / n} \leq \lambda^{-(1 / 2)}
$$

Since

$$
\left\|f_{*}^{-n}\left|\Sigma^{b}\left(E^{1}\right)\|\geq\| T f^{-n}\right| E_{p}^{1}\right\| \quad \text { for all } p \in \Lambda,
$$

we have

$$
\left\|T f^{-n} \mid E_{f^{n}(p)}^{1}\right\| \leq\left(\lambda^{-1}\right)^{n / 2} \quad \text { for all } p \in \Lambda .
$$

Combining with (2.3) (ii), we get the lemma.

Put $E=T M \mid \Lambda=E^{1} \oplus E^{2}$ and we regard $E$ as a vector bundle over $E^{1}$ by projecting each fiber onto $E^{1}$ along $E^{2}$. Let

$$
\Sigma\left(E^{1}, E\right)=\left\{\text { continuous sections } E^{1} \rightarrow E\right\} .
$$

Define a metric on $\Sigma\left(E^{1}, E\right)$ by

$$
\left\|\sigma-\sigma^{\prime}\right\|_{*}=\sup _{p \in A} \sup _{\substack{x \in E^{1} \\ x \neq 0}} \frac{\left|\sigma x-\sigma^{\prime} x\right|}{|x|} .
$$

Let

$$
\Sigma_{0}=\left\{\sigma \in \Sigma\left(E^{1}, E\right):\|\sigma\|_{*}<\infty\right\}
$$

and

$$
\Sigma(1 / 2)=\left\{\sigma \in \Sigma_{0}: \sigma_{p}(0)=0, L\left(\sigma_{p}\right) \leq 1 / 2 \text { for all } p \in \Lambda\right\}
$$

where $\sigma_{p}: E_{p}^{1} \rightarrow E_{p}^{2}$ is defined by

$$
\left(\sigma \mid E_{p}^{1}\right)(x)=\left(x, \sigma_{p}(x)\right) \in E_{p}^{1} \oplus E_{p}^{2} \quad \text { for } x \in E_{p}^{1},
$$

and $L\left(\sigma_{p}\right)$ denotes the Lipschitz constant of $\sigma_{p}$. With the metric $\|\cdot\|_{*}, \Sigma_{0}$ is a Banach space and $\Sigma(1 / 2)$ is a closed subset (ref. (5.2) in [4]).

We define $\tilde{f}: T M|\Lambda \rightarrow T M| \Lambda$ by $\exp _{f(p)}^{-1} f \exp _{p}$ on a neighborhood of 0 in $T_{p} M$ for each $p \in \Lambda$, and extend it to all over $T M \mid \Lambda$ by combining with $T f$ by a smooth bump function. Note that $\sup _{p \in \Lambda} L\left((\tilde{f}-T f) \mid T_{p} M\right)$ can be forced as small as desired by restricting $\tilde{f}$ to a smaller neighborhood of 0 before averaging with $T f$.

Let $N \in Z_{+}$satisfy $N \geq n_{0}$ and $c\left(\lambda^{1 / 2}\right)^{N}<1$, and put $g=f^{N}$. Then from (5.1), there is a $0<\rho<1$ such that

$$
\left\|T g\left|E_{p}^{2}\|\cdot\| T g^{-1}\right| E_{g(p)}^{1}\right\|^{k} \leq \rho
$$

for all $p \in \Lambda$ and $k=1,2$. 
For any $\sigma \in \Sigma(1 / 2)$, we define

$$
g_{\sharp} \sigma=\tilde{g} \sigma h, \quad h=\left(\pi_{1} \tilde{g} \sigma\right)^{-1},
$$

where $\tilde{g}=f^{N}$ and $\pi_{1}: E \rightarrow E^{1}$ is the canonical projection. By the almost same estimates as in [4], we can see that $g_{\#}$ is well-defined and a contraction on $\Sigma(1 / 2)$. This gives a unique invariant section $\sigma^{1}$ which satisfy (5.2) For all $p \in \Lambda$,

(i ) $L\left(\sigma_{p}^{1}\right) \leq 1 / 2$

(ii) $\sigma_{p}^{1}(0)=0$

(iii) $\sigma_{p}^{1}: E_{p}^{1} \rightarrow E_{p}^{2}$ is of class $C^{2}$

(iv) $T_{0} \sigma_{p}^{1}=0$

(v) $\tilde{g}\left(\operatorname{graph}\left(\sigma_{p}^{1}\right)\right)=\operatorname{graph}\left(\sigma_{p}^{1}\right)$

(vi) $\sigma_{p}^{1}: E_{p}^{1} \rightarrow E_{p}^{2}$ depends continuously on $p \in \Lambda$ as $C^{2}$ map.

Put $W_{p}^{1}(r)=\exp _{p}\left(\operatorname{graph}\left(\sigma_{p}^{1}\right) \cap V_{p}(r)\right)$ for $r>0$, where

$$
V_{p}(r)=\left\{\left(v_{1}, v_{2}\right) \in E_{p}^{1} \oplus E_{p}^{2}:\left|V_{1}\right| \leq r,\left|v_{2}\right| \leq r\right\} .
$$

$\left\{W_{p}^{1}(r)\right\}_{p \in A}$ is called locally $g$-invariant $C^{2}$ pre-limination for small $r>0$ (ref. (5.5) in [4]).

Now we shall show that by taking $\sup _{p \in \Lambda} L\left((\tilde{f}-T f) \mid T_{p} M\right)$ and $r>0$ small, $\left\{W_{p}^{1}(r)\right\}_{p \in A}$ is $f$-invariant.

Recall that $g=f^{N}$, and $N \in Z_{+}$satisfy $N \geq n_{0}$ and $c\left(\lambda^{1 / 2}\right)^{N} \leq \rho<1$. Consider $g_{1}=f^{N}, g_{2}=f^{N+1}$ and $g_{3}=f^{N(N+1)}$. As above, they satisfy

$$
\left\|T g_{i}\left|E_{p}^{2}\|\cdot\| T g_{i}^{-1}\right| E_{g_{i}(p)}^{1}\right\|^{k} \leq \rho<1
$$

for all $p \in \Lambda, k=1,2$. Therefore,

$$
g_{i \sharp}: \Sigma(1 / 2) \longrightarrow \Sigma(1 / 2)
$$

has a unique fixed point $\sigma_{i}$ for $i=1,2,3$. Taking $\sup _{p \in \Lambda} L\left((\tilde{f}-T f) \mid T_{p} M\right)$ sufficiently small, by induction, we can see that,

(i ) $\left(g_{1 \#}\right)^{N+1}=g_{3 \#}=\left(g_{2 \sharp}\right)^{N}$ on $\Sigma(1 / 2)$

(ii) $f_{\sharp}: \Sigma(1 / 2) \rightarrow \Sigma_{0}$ is well-defined by $f_{\sharp} \sigma=\tilde{f} \sigma h$, where $h=\left(\pi_{1} \tilde{f} \sigma\right)^{-1}$

and has the property that

$$
g_{2 \sharp}=f_{\sharp} \circ g_{1 \sharp} \quad \text { on } \Sigma(1 / 2) \text {. }
$$

From (i), we get $\sigma_{1}=\sigma_{2}=\sigma_{3}$. Denoting this $\sigma^{1}$, from (ii), we have 


$$
\sigma^{1}=g_{2 \sharp} \sigma^{1}=f_{\#} g_{1 \sharp} \sigma^{1}=f_{\sharp} \sigma^{1} \text {. }
$$

This implies that

$$
\tilde{f}\left(\operatorname{graph}\left(\sigma^{1}\right)\right)=\operatorname{graph}\left(\sigma^{1}\right) .
$$

Similarly, there is a $\tilde{f}$-invariant section

$$
\sigma^{2}: E^{2} \longrightarrow E^{1} \oplus E^{2}=E=T M \mid \Lambda,
$$

satisfying the similar property to (5.2).

Now we are ready to give the precise definition of the "Semi-invariant coordinate for $f$ ". "Semi-invariant" means the invariance of $x$ and $y$-axes by $f$.

For each $p \in \Lambda$, we define a $C^{2}$ diffeomorphism $a_{p}: T_{p} M \rightarrow T_{p} M$ by

$$
a_{p}\left(v_{1}, v_{2}\right)=\left(v_{1}-\sigma_{p}^{2}\left(v_{2}\right), v_{2}-\sigma_{p}^{1}\left(v_{1}\right)\right)
$$

for $v_{i} \in E_{p}^{i}$.

By the properties of $\sigma^{i}$, we can see that $a_{p}$ satisfy

(5.3) For all $p \in \Lambda$,

(i ) $a_{p}$ is a $C^{2}$ diffeomorphism

(ii) $a_{p}(0)=0$ and $T_{0} a_{p}=$ id

(iii) $a_{p}\left(\operatorname{graph}\left(\sigma_{p}^{1}\right)\right)=E_{p}^{1} \oplus\{0\}$

$a_{p}\left(\operatorname{graph}\left(\sigma_{p}^{2}\right)\right)=\{0\} \oplus E_{p}^{2}$.

Remark. The properties $L\left(\sigma_{p}^{1}\right) \leq 1 / 2, L\left(\sigma_{p}^{2}\right) \leq 1 / 2$ are used to prove (i).

Let $p \in \Lambda$ and $e_{p}^{i}$ be a unit vector in $E_{p}^{i}$. Define an isomorphism $d_{p}: T_{p} M=E_{p}^{1} \oplus E_{p}^{2} \rightarrow R^{2}$ by

$$
d_{p}\left(c_{1} e_{1}+c_{2} e_{2}\right)=\left(c_{1}, c_{2}\right), \quad c_{i} \in \boldsymbol{R} .
$$

Remark that this definition of $d_{p}$ depends on the choice of the basis of $E_{p}^{1} \oplus E_{p}^{2}=T_{p} M$.

For small $r>0$ and each $p \in \Lambda$, we define a $C^{2}$ local coordinate $\psi_{p}:\left(U_{p}(r), p\right) \rightarrow\left(\boldsymbol{R}^{2}, 0\right)$ by

$$
\psi_{p}=d_{p} \circ a_{p} \circ \exp _{p}^{-1},
$$

where $U_{p}(r)$ denotes the $r$-neighborhood of $p$.

$\psi_{p}$ has the property that,

$$
\begin{aligned}
& \psi_{p}\left(W_{p}^{1}\left(r^{\prime}\right)\right) \subset\{x \text {-axis }\} \\
& \psi_{p}\left(W_{p}^{2}\left(r^{\prime}\right)\right) \subset\{y \text {-axis }\}
\end{aligned}
$$

for small $r^{\prime}>0$ and $p \in \Lambda$. 
Put $f_{p}=\psi_{f(p)} \circ f \circ \psi_{p}^{-1}$ for $p \in \Lambda$.

From the above argument, we can see that,

(5.4) There exist $r_{1}>0$ and $r_{2}>0$ with the following properties.

(i ) $\psi_{p}^{-1}:\left(U\left(r_{1}\right), 0\right) \rightarrow(M, p)$ is a well-defined $C^{2}$-diffeomorphism for all $p \in \Lambda$, where $U\left(r_{1}\right)$ is the $r_{1}$-neighborhood of the origin in $R^{2}$.

(ii) $f_{p}:\left(U\left(r_{1}\right), 0\right) \rightarrow\left(\boldsymbol{R}^{2}, 0\right)$ is well-defined and preserves $x$ and $y$-axes, for all $p \in \Lambda$.

(iii) for $p, q \in \Lambda$, if $d(p, q)<r_{2}$ then $\psi_{p} \psi_{q}^{-1}: U\left(r_{1}\right) \rightarrow R^{2}$ is a well-defined $C^{2}$ diffeomorphism.

(iv) for any $\varepsilon>0$, there exists $0<\delta<r_{2}$ such that if $d(p, q)<\delta$ then $\left\|\psi_{p} \psi_{q}^{-1}-\mathrm{id}\right\|_{2}<\varepsilon$ on $U\left(r_{1}\right)$, where $\psi_{p}$ and $\psi_{q}$ are defined with the bases which are near each other, and $\|\cdot\|_{2}$ denotes the $C^{2}$-norm for functions on $\boldsymbol{R}^{2}$.

$$
\sup _{\substack{p \in A_{1, x \in U\left(r_{1}\right)} \\ i, j, k=1,2}}\left|\frac{\partial^{2} f_{p}^{k}}{\partial x_{i} \partial x_{j}}(x)\right|=M_{0}<\infty
$$

where $f_{p}=\left(f_{p}^{1}, f_{p}^{2}\right)$.

(vi) there is $c_{1}>0$ such that, for any measurable subset $A$ of $U\left(r_{1}\right)$,

$$
c_{1} s(A) \leq s\left(\psi_{p}^{-1}(A)\right) \leq c_{1}^{-1} s(A)
$$

for all $p \in \Lambda$, where $s(A)$ denotes the measure (area) of $A$.

From the definition of $\psi_{p}$, we get

$$
\left\|T f\left|E_{p}^{i} \|=\right| \frac{\partial f_{p}^{i}}{\partial x_{i}}(0,0) \mid \quad \text { for all } p \in \Lambda \text {, and } i=1,2\right. \text {. }
$$

Regard $f_{p}^{1}(x, y)$ as a function of $x$. Applying the Taylor expansion up to 2 nd order around $(0, y)$, we have,

$$
f_{p}^{1}(x, y)=f_{p}^{1}(0, y)+x \cdot \frac{\partial f_{p}^{1}}{\partial x}(0, y)+\frac{x^{2}}{2} \cdot \frac{\partial^{2} f_{p}^{1}}{\partial x^{2}}(\theta x, y),
$$

for some $0 \leq \theta \leq 1$. By the Mean value theorem,

$$
\frac{\partial f_{p}^{1}}{\partial x}(0, y)=\frac{\partial f_{p}^{1}}{\partial x}(0,0)+y \cdot \frac{\partial^{2} f_{p}^{1}}{\partial x \partial y}\left(0, \theta^{\prime} y\right)
$$

for some $0 \leq \theta^{\prime} \leq 1$. Since $f_{p}$ preserves $y$-axis, $f_{p}^{1}(0, y)=0$. Thus, we get

$$
\left|f_{p}^{1}(x, y)\right| \leq|x| \cdot\left\{\left|\frac{\partial f_{p}^{1}}{\partial x}(0,0)\right|+M_{0}(|x|+|y|)\right\} .
$$

Similarly, 


$$
\left|f_{p}^{2}(x, y)\right| \leq|y| \cdot\left\{\left|\frac{\partial f_{p}^{2}}{\partial y}(0,0)\right|+M_{0}(|x|+|y|)\right\}
$$

Put

$$
m_{0}=\inf _{\substack{p \in \Lambda \\ i=1,2}}\left\|T f \mid E_{p}^{i}\right\| \quad \text { and } \quad M_{1}=M_{0} / m_{0} .
$$

Then, from (5.5), we have

$$
\begin{aligned}
& \left|f_{p}^{1}(x, y)\right| \leq|x| \cdot\left\|T f \mid E_{p}^{1}\right\| \cdot\left\{1+M_{1}(|x|+|y|)\right\} \\
& \left|f_{p}^{2}(x, y)\right| \leq|y| \cdot\left\|T f \mid E_{p}^{1}\right\| \cdot\left\{1+M_{1}(|x|+|y|)\right\}
\end{aligned}
$$

for all $p \in \Lambda$ and $(x, y) \in U\left(r_{1}\right)$.

\section{§6. Tracing boxes}

In this section, we shall define the box neighborhoods for each $p \in \Lambda$ with respect to the semi-invariant coordinate, and see how the iterated images of these boxes behave.

Put

$$
B\left(r^{\prime}, r^{\prime \prime}\right)=\left\{(x, y) \in R^{2}:|x| \leq r^{\prime},|y| \leq r^{\prime \prime}\right\}
$$

and

$$
B(r)=B(r, r) .
$$

For $p \in \Lambda$ and $0<r, r^{\prime}, r^{\prime \prime}<r_{1} / 2$, we define $\left(p, r^{\prime}, r^{\prime \prime}\right)$-box $B_{p}\left(r^{\prime}, r^{\prime \prime}\right)$ by

$$
B_{p}\left(r^{\prime}, r^{\prime \prime}\right)=\psi_{p}^{-1}\left(B\left(r^{\prime}, r^{\prime \prime}\right)\right)
$$

and $(p, r)$-box $B_{p}(r)$ by

$$
B_{p}(r)=\psi_{p}^{-1}(B(r)) .
$$

Definition. Let $p \in \Lambda, n \in Z_{+}$and $\delta>0 . \quad B_{p}\left(r^{\prime}, r^{\prime \prime}\right)$ is $(p, n, \delta)$-tracing box if and only if

$$
f^{k}\left(B_{p}\left(r^{\prime}, r^{\prime \prime}\right)\right) \subset B_{f^{k}(p)}(\delta) \quad \text { for all } 0 \leq k \leq n .
$$

Remark. While the definition of $\psi_{p}$ depends on the choice of the basis of $E_{p}^{1} \oplus E_{p}^{2}$, the set $B_{p}\left(r^{\prime}, r^{\prime \prime}\right)$ does not, because $B\left(r^{\prime}, r^{\prime \prime}\right)$ is symmetric with respect to $x$ and $y$-axes respectively.

Two near boxes are related in the following way.

(6.1) Lemma. There exists $r_{3}>0$ such that, if $p, q \in \Lambda$ and $B_{p}(r) \cap$ $B_{q}\left(r^{\prime}\right) \neq \phi$ for $0<r, r^{\prime}<r_{3}$, then 
(i) $q \in B_{p}\left(r+2 r^{\prime}\right)$

(ii) $B_{q}\left(r^{\prime}\right) \subset B_{p}\left(r+4 r^{\prime}\right)$.

Proof. Assume that $B_{p}(r) \cap B_{q}\left(r^{\prime}\right) \neq \phi$, that is;

$$
B(r) \cap \psi_{p} \psi_{q}^{-1} B\left(r^{\prime}\right) \neq \phi .
$$

From the above remark, $B_{q}\left(r^{\prime}\right)$ does not depend on the choice of the basis with respect to which $\psi_{q}$ is defined. So, we can assume that $\psi_{p}$ and $\psi_{q}$ are defined by the bases which are near each other. Choose 0 $<\delta<r_{2}$ as in (5.4) (iv) with $\varepsilon=1 / 2$. Take $r_{3} \leq \delta$. Then for $x \in B\left(r^{\prime}\right)$ with $r^{\prime}<r_{3}$, we have from (5.4) (iv),

$$
\begin{aligned}
& \left|\psi_{p} \psi_{q}^{-1}(x)-\psi_{p} \psi_{q}^{-1}(0)-x\right| \\
& \quad=\left|\left(\psi_{p} \psi_{q}^{-1}-\mathrm{id}\right)(x)-\left(\psi_{p} \psi_{q}^{-1}-\mathrm{id}\right)(0)\right| \\
& \quad \leq\left\{\sup _{y \in B\left(r^{\prime}\right)}\left\|T_{y}\left(\psi_{p} \psi_{q}^{-1}-\mathrm{id}\right)\right\|\right\} \cdot|x| \\
& \quad \leq|x| / 2 \leq \sqrt{2}\left(r^{\prime} / 2\right) .
\end{aligned}
$$

So, for $i=1,2$,

$$
\left|\left(\psi_{p} \psi_{q}^{-1}(x)\right)_{i}-\left(\psi_{p} \psi_{q}^{-1}(0)\right)_{i}\right| \leq \sqrt{2}\left(r^{\prime} / 2\right)+\left|x_{i}\right|<2 r^{\prime},
$$

where $x=\left(x_{1}, x_{2}\right)$ and ()$_{i}$ denotes the $i$-th coordinate.

Take a point $x_{0} \in B(r) \cap \psi_{p} \psi_{q}^{-1} B\left(r^{\prime}\right)$ and put $x=\left(\psi_{p} \psi_{q}^{-1}\right)^{-1}\left(x_{0}\right) \in B\left(r^{\prime}\right)$. Then, since $q=\psi_{q}^{-1}(0)$ and $\psi_{p} \psi_{q}^{-}(x) \in B(r)$,

$$
\begin{aligned}
\left|\left(\psi_{p}(q)\right)_{i}\right| & \leq\left|\left(\psi_{p} \psi_{q}^{-1}(x)\right)_{i}-\left(\psi_{p}(q)\right)_{i}\right|+\left|\left(\psi_{p} \psi_{q}^{-}(x)\right)_{i}\right| \\
& <2 r^{\prime}+r
\end{aligned}
$$

This implies (i). A similar argument gives (ii).

Let $p \in \Lambda, n \in Z_{+}$and $N>0$ (possibly not integer). For the sake of convenience, we put

$$
\begin{aligned}
& \theta(p, n)=\left\|T f^{n} \mid E_{p}^{1}\right\| \\
& \omega(p, n)=\left\|T f^{n} \mid E_{p}^{2}\right\| \\
& \eta(p, N)=\max _{0 \leq k \leq[N]}\left\|T f^{k} \mid E_{p}^{1}\right\| .
\end{aligned}
$$

Remark that, since $\operatorname{dim} E^{1}=\operatorname{dim} E^{2}=1$,

$$
\begin{aligned}
& \theta(p, n+m)=\theta(p, n) \cdot \theta\left(f^{n}(p), m\right) \\
& \omega(p, n+m)=\omega(p, n) \cdot \omega\left(f^{n}(p), m\right)
\end{aligned}
$$

for any $p \in \Lambda$ and $n, m \in \boldsymbol{Z}_{+}$. 
The iterated images of the box neighborhoods behave as follows.

(6.2) Lemma. Let $p \in \Lambda, N \geq 1$ (possibly not integer), $K \in Z_{+}$and $r_{4}=$ $\min \left\{r_{1} / 2 c, 1 / M_{1}(1+c)\right\}$. If $\rho \geq \eta(p, K)$ and $0<r \leq r_{4}$, then

$$
f^{i}\left(B_{p}(r / \rho N)\right) \subset B_{f^{i}(p)}(r \theta(p, i) / \rho(N-i), r \omega(p, i) / \rho(N-i))
$$

for all integers $0 \leq i \leq \min \{K, N-1\}$.

Proof. Since $\rho \geq \eta(p, K) \geq \theta(p, i)$ and $N-i \geq 1$ for all integers $0 \leq$ $i \leq \min \{K, N-1\}$, we have $\theta(p, i) / \rho(N-i) \leq 1$. From (2.3) (ii), $\omega(p, i)$ $\leq c \theta(p, i)$. Then since $r \leq r_{4}$, we have

$$
\begin{aligned}
& r \theta(p, i) / \rho(N-i) \leq r_{1} / 2 c \\
& r \omega(p, i) / \rho(N-i) \leq r_{1} / 2 .
\end{aligned}
$$

This implies that $B_{f^{i}(p)}(r \theta(p, i) / \rho(N-i), r \omega(p, i) / \rho(N-i))$ is well-defined for all integers $0 \leq i \leq \min \{K, N-1\}$.

We shall prove the lemma by induction. For $i=0$, it is trivial. To simplify notations, we put

$$
B_{j}=B_{f^{j}(p)}(r \theta(p, j) / \rho(N-j), r \omega(p, j) /(N-j))
$$

for all integers $0 \leq j \leq \min \{K, N-1\}$.

Suppose that the lemma is true for $i-1$. Then

$$
f^{i-1}\left(B_{p}(r / \rho N)\right) \subset B_{i-1} \text {. }
$$

It suffices to show that $f\left(B_{i-1}\right) \subset B_{i}$. Namely we shall see that for $(x, y)$ $=\psi_{f^{i-1(p)}}(z)$ and $z \in B_{i-1}$, we have

$$
\begin{aligned}
& r \theta(p, i) / \rho(N-i) \geq\left|f_{1}(x, y)\right| \\
& r \omega(p, i) / \rho(N-i) \geq\left|f_{2}(x, y)\right|
\end{aligned}
$$

where $\left(f_{1}, f_{2}\right)=\psi_{f^{i}(p)} \circ f \circ \psi_{f^{i-1}(p)}^{-1}$. In fact, from (5.6),

$$
\begin{aligned}
& r \theta(p, i) / \rho(N-i)-\left|f_{1}(x, y)\right| \\
& \geq\{r \theta(p, i) / \rho(N-i)\}-|x| \cdot\left\|T f \mid E_{f^{i-1}(p)}^{1}\right\| \cdot\left\{1+M_{1}(|x|+|y|)\right\} \\
& \geq\{r \theta(p, i) / \rho(N-i)\}-\{r \theta(p, i) / \rho(N-i+1)\} \\
& \cdot\left\{1+M_{1} r(\theta(p, i-1)+\omega(p, i-1)) / \rho(N-i+1)\right\} \\
&=\{r \theta(p, i) / \rho\} \cdot\left\{1 / \rho(N-i+1)^{2}(N-i)\right\} \\
& \cdot\left\{(N-i+1)\left(\rho-M_{1} r(\theta(p, i-1)+\omega(p, i-1))\right)\right. \\
&\left.+M_{1} r(\theta(p, i-1)+\omega(p, i-1))\right\} .
\end{aligned}
$$


Thus, we only need to prove $\rho-M_{1} r(\theta(p, i-1)+\omega(p, i-1)) \geq 0$. In fact, we get

$$
\begin{aligned}
\rho-M_{1} r(\theta+\omega) & \geq \rho-M_{1} r(\theta+c \theta) \\
& =\rho\left\{1-M_{1} r \theta(p, i-1)(1+c) / \rho\right\} \\
& \geq \rho\left\{1-M_{1} r(1+c)\right\} \geq 0,
\end{aligned}
$$

because $\omega(p, i-1) \leq c \theta(p, i-1), \rho \geq \theta(p, i-1)$ and $r \leq 1 / M_{1}(1+c)$.

Similarly $r \omega(p, i) / \rho(N-i)-\left|f_{2}(x, y)\right| \geq 0$. This completes the proof.

By this lemma and (2.3) (ii), the next lemma is easily proved.

(6.3) LEMmA. Under the same hypothesis as in (6.2),

$$
f^{i}\left(B_{p}(r / \rho N)\right) \subset B_{f i(p)}(\operatorname{cr} \theta(p, i) / \rho(N-i))
$$

for all integers $0 \leq i \leq \min \{K, N-1\}$.

This lemma says that the box $B_{p}(r / \rho N)$ is a $(p, \min \{K, N-1\}, c r)$ tracing box. A more useful modification of (6.2) is the following,

(6.4) Lemma. Under the same hypothesis as in (6.2),

$$
f^{j}\left(B_{f^{i}(p)}(r \theta(p, i) / \rho(N-i))\right) \subset B_{f^{i+j}(p)}(\operatorname{cr} \theta(p, i+j) / \rho(N-i-j))
$$

for all integers $i, j$ satisfying $0 \leq i+j \leq \min \{K, N-1\}$.

Proof.

$$
\begin{aligned}
\eta\left(f^{i}(p), K-i\right) & =\max _{0 \leq n \leq K-i}\left\|T f^{n} \mid E_{f^{i}(p)}^{1}\right\| \\
& =\max _{0 \leq n \leq K-i}\left\{\left\|T f^{n+i}\left|E_{p}^{1}\|/\| T f^{i}\right| E_{p}^{1}\right\|\right\} \\
& \leq \eta(p, K) / \theta(p, i) \leq \rho / \theta(p, i) .
\end{aligned}
$$

From (6.3), we have

$$
\begin{aligned}
& f^{j}\left(B_{f^{i}(p)}(r \theta(p, i) / \rho(N-i))\right) \\
& \quad=f^{j}\left(B_{f^{i}(p)}(r /(\rho / \theta(p, i))(N-i))\right) \\
& \quad \subset B_{f^{i+j(p)}}\left(\operatorname{cr} \theta\left(f^{i}(p), j\right) /(\rho / \theta(p, i))(N-i-j)\right) \\
& \quad=B_{f^{i+j(p)}}(\operatorname{cr} \theta(p, i+j) / \rho(N-i-j)) .
\end{aligned}
$$

This implies the lemma.

\section{§7. The fundamental lemma for the closing lemma}

In this and the next sections we shall formulate "the closing lemma for $F(M)$ " which plays an essential role in the proof of our theorem. 
In the general case, "The $C^{1}$ closing lemma" has been established by C. Pugh and C. Robinson in [12], and here we just adapt their method to our situation. In our case, $\operatorname{dim} M=2$ and we are given a $T f$-invariant splitting $T M \mid \Lambda=E^{1} \oplus E^{2}$, so we don't need the "Linear algebra" which complicates the argument of [12]. On the other hand, we will need delicate perturbations of $f$ in the succeeding sections, so we must formulate the closing lemma precisely for our purpose.

In this section, we shall prove the fundamental lemma by which we can select a pair of points that are particularly well situated regarding other points considered. The proof is a fairly easy analogy of (4.2) Fundamental Lemma in [12] in terms of our semi-invariant coordinate.

To simplify computations, we use the following norm on $T M \mid \Lambda$.

$$
|v|=\left(\left|v_{1}\right|^{2}+\left|v_{2}\right|^{2}\right)^{1 / 2}
$$

where $v \in T_{p} M, p \in \Lambda$ and $v=v_{1}+v_{2} \in E_{p}^{1} \oplus E_{p}^{2}$. Clearly this norm is equivalent to the norm defined by the Riemannian metric.

Before formulating the Fundamental lemma, we need the following technical lemma.

(7.1) Lemma. For any $\varepsilon>0$, there exists $\delta>0$ such that

$$
\sup _{p \in \Lambda, x \in V_{p}(\hat{o})}\left\{\left\|T_{x}\left(a_{p}-\mathrm{id}\right)\right\|,\left\|T_{x}\left(a_{p}^{-1}-\mathrm{id}\right)\right\|\right\} \leq \varepsilon .
$$

The proof is straightforward by using (5.2) (vi), (5.3) (ii) and compactness of $\Lambda$.

Let $p \in M, v \in T_{p} M$ and $A: T_{p} M \rightarrow T_{p} M$ be linear. We define a map $b_{p}(v, A):\left\{\right.$ a neighborhood of 0 in $\left.T_{p} M\right\} \rightarrow M$ by

$$
b_{p}(v, A)(x)=\exp _{p}(v+A x) \quad \text { for } x \in T_{p} M .
$$

Especially, for $p \in \Lambda$ and positive numbers $a_{1}, a_{2}$, we define

$$
b_{p}\left(v, a_{1}, a_{2}\right)=b_{p}\left(v, A\left(a_{1}, a_{2}\right)\right)
$$

where $A\left(a_{1}, a_{2}\right)\left(v_{1}+v_{2}\right)=a_{1} v_{1}+a_{2} v_{2}$ for $v_{i} \in E_{p}^{i}$.

Let $\delta_{0}>0$ be given in (7.1) with $\varepsilon=1 / 8$. Define a number, $r_{5}=$ $\min \left\{r_{1} / 60, \delta_{0} / 60\right\}$.

(7.2) Lemma (Fundamental lemma). Let $\left\{p_{n}\right\}$ be a finite subset of $M$, $0<r<r_{5}$ and $p \in \Lambda$. If there exist two points $p_{i}, p_{j} \in\left\{p_{n}\right\}$ satisfying $p_{i}, p_{j}$ $\in B_{p}(r)$, then there exist two points $p_{s}, p_{t} \in\left\{p_{n}\right\}, v \in T_{p} M$ and positive numbers $a_{1}, a_{2}$ such that 
(i) $|v|+a_{1}+a_{2} \leq 30 r$

(ii) $(2 \sqrt{2})^{-1} \leq a_{1} / a_{2} \leq 2 \sqrt{2}$

(iii) $b_{p}\left(v, a_{1}, a_{2}\right)\left(V_{p}(1)\right) \subset B_{p}(30 r)$

(iv) $p_{s}, p_{t} \in b_{p}\left(v, a_{1}, a_{2}\right)\left(V_{p}(\sqrt{3 / 4})\right)$

(v) $p_{k} \notin b_{p}\left(v, a_{1}, a_{2}\right)\left(V_{p}(1)\right)$ for $p_{k} \in\left\{p_{n}\right\}$ with $p_{k} \neq p_{s}, p_{t}$.

Proof. Suppose that $p_{i}, p_{j} \in B_{p}(r)$. For $p_{n}$ near $p$, we put $\tilde{p}_{n}=$ $\exp _{p}^{-1} p_{n}$. Remark that we don't need to consider the points that are far from $p$. Put $x_{0}=\tilde{p}_{i}, y_{0}=\tilde{p}_{j}$ and we shall select a sequence $\left(x_{n}, y_{n}\right)$ of the points of $\left\{\tilde{p}_{n}\right\}$ as follows. Define

$$
\xi(x, y)=\left\{z \in T_{p} M:|x-z| \leq \sqrt{3 / 4}|x-y| \text { or }|y-z| \leq \sqrt{3 / 4}|x-y|\right\}
$$

for $x, y \in T_{p} M$. If some point $z \in\left\{\tilde{p}_{n}\right\}$ is contained in $\xi\left(x_{0}, y_{0}\right)$, let

$$
\begin{array}{ll}
z \text { replace } y_{0} & \text { if }\left|z-x_{0}\right| \leq\left|z-y_{0}\right| \\
z \text { replace } x_{0} & \text { if }\left|z-y_{0}\right| \leq\left|z-x_{0}\right| .
\end{array}
$$

Let $\left(x_{1}, y_{1}\right)$ be the pair so formed. Proceed as with $\left(x_{0}, y_{0}\right)$, generating a sequence $\left(x_{n}, y_{n}\right)$. This process ends at finite steps, because

$$
\left|x_{n}-y_{n}\right| \leq(\sqrt{3 / 4})^{n}\left|x_{0}-y_{0}\right|
$$

and $\left\{\tilde{p}_{n}\right\}$ is a finite set. Let $\left(x_{k}, y_{k}\right)=\left(\tilde{p}_{s}, \tilde{p}_{t}\right)$ be the final pair. This has the property that no other point of $\left\{\tilde{p}_{n}\right\}$ is contained in $\xi\left(x_{k}, y_{k}\right)$. Also we can see that

$$
\left|x_{k}-x_{0}\right| \leq \sum_{n=1}^{k}\left|x_{n}-x_{n-1}\right| \leq\left\{\sum_{n=1}^{k}(3 / 4)^{n / 2}\right\}\left|x_{0}-y_{0}\right|
$$

because either $x_{n}=x_{n-1}$ or $y_{n}=y_{n-1}$, and

$$
\left|x_{n}-x_{n-1}\right| \leq \sqrt{3} \overline{3}\left|x_{n-1}-y_{n-1}\right| \text {. }
$$

Now set

$$
v=(1 / 2)\left(\tilde{p}_{s}+\tilde{p}_{t}\right)
$$

and

$$
a_{i}=\left((1 / 3)\left|\tilde{p}_{s}-\tilde{p}_{t}\right|_{i}^{2}+(1 / 16)\left|\tilde{p}_{s}-\tilde{p}_{t}\right|^{2}\right)^{1 / 2} \quad \text { for } i=1,2
$$

where $|u|_{i}=\left|u_{i}\right|$ for $u=u_{1}+u_{2} \in E_{p}^{1} \oplus E_{p}^{2}=T_{p} M$.

By easy calculations, we have

$$
\begin{aligned}
& v+A\left(a_{1}, a_{2}\right)\left(V_{p}(1)\right) \subset \xi\left(\tilde{p}_{s}, \tilde{p}_{t}\right) \\
& \tilde{p}_{s}, \tilde{p}_{t} \in v+A\left(a_{1}, a_{2}\right)\left(V_{p}(\sqrt{ } 3 / 4)\right)
\end{aligned}
$$




$$
(2 \sqrt{2})^{-1} \leq a_{1} / a_{2} \leq 2 \sqrt{2} .
$$

It remains to show (i) and (iii). Since $\psi_{p}\left(p_{i}\right) \in B(r)$, we have $d_{p}^{-1} \psi_{p}\left(p_{i}\right) \epsilon$ $V_{p}(r)$. Therefore

$$
\begin{aligned}
\left|\tilde{p}_{i}\right| & =\left|\exp _{p}^{-1} p_{i}\right|=\left|a_{p}^{-1} d_{p}^{-1} \psi_{p} p_{i}\right| \\
& \leq\left|\left(a_{p}^{-1}-\mathrm{id}\right)\left(d_{p}^{-1} \psi_{p}\left(p_{i}\right)\right)\right|+\left|d_{p}^{-1} \psi_{p}\left(p_{i}\right)\right| \\
& \leq \sqrt{2} r / 8+\sqrt{2} r=(9 \sqrt{2} / 8) r .
\end{aligned}
$$

Similarly $\left|\tilde{p}_{j}\right| \leq(9 \sqrt{2} / 8) r$. By using (1), (2) and (7.1), we can show that

$$
|v|+a_{1}+a_{2} \leq 30 r
$$

and

$$
v+A\left(a_{1}, a_{2}\right)\left(V_{p}(1)\right) \subset a_{p}^{-1} d_{p}^{-1} B(30 r) .
$$

This completes the lemma.

To simplify notations in the succeeding sections, we introduce the following definition.

(7.3) Definition. Let $p \in \Lambda$ and $r>0$. Let $x, y \in M, v \in T_{p} M$ and $a_{1}, a_{2}$ be positive numbers. A quintuple $\left(x, y, v, a_{1}, a_{2}\right)$ is $(p, r)$-connectable if and only if

(i) $|v|+a_{1}+a_{2} \leq r$

(ii) $(2 \sqrt{2})^{-1} \leq a_{1} / a_{2} \leq 2 \sqrt{2}$

(iii) $x, y \in b_{p}\left(v, a_{1}, a_{2}\right)\left(V_{p}(\sqrt{3 / 4})\right)$

(iv) $b_{p}\left(v, a_{1}, a_{2}\right)\left(V_{p}(1)\right) \subset B_{p}(r)$.

With this definition, we can formulate (7.2) as follows.

(7.4) Lemma. Let $\left\{p_{n}\right\}$ be a finite subset of $M, 0<r<r_{5}$ and $p \in \Lambda$. If there are two points $p_{i}, p_{j} \in B_{p}(r)$, then there exist $v \in T_{p} M$, positive numbers $a_{1}, a_{2}$ and two points $p_{s}, p_{t}$ in $\left\{p_{n}\right\}$ such that $\left(p_{s}, p_{t}, v, a_{1}, a_{2}\right)$ is $(p, 30 r)$-connectable and moreover $p_{k} \neq b_{p}\left(v, a_{1}, a_{2}\right)\left(V_{p}(1)\right)$ for $p_{k} \in\left\{p_{n}\right\}$ with $p_{k} \neq p_{s}, p_{t}$.

\section{§8. Closing lemma for $F(M)$}

In this section, we shall give a precise formulation of the "Closing lemma for $F(M)$ ".

First, by an entire analogy with [12], using $T M \mid \Lambda=E^{1} \oplus E^{2}$ and (2.3) (ii) instead of $V^{1} \oplus V^{2}$ and hyp $\left(T f^{n}\right.$ : resp. $\left.V^{1} \oplus V^{2}\right)$ in [12], and perturbing $f$ around the points $\left\{f^{n}\left(p_{*}\right)\right\}_{-N \leq n \leq N-1}$, we get the following; 
(8.1) Lemma. For any $C^{1}$-neighborhood $\mathscr{U}$ of $f$, there exist $r>0$ and $N \in Z_{+}$with the following property. Let $p_{*} \in \Lambda$ and $p, q \in M$. If $v \in T_{p_{*}} M$ and positive numbers $a_{1}, a_{2}$ satisfy

(I ) $|v|+a_{1}+a_{2} \leq r$

(II) $(2 \sqrt{2})^{-1} \leq a_{1} / a_{2} \leq 2 \sqrt{2}$

(III) $f^{n}\left(b_{p_{*}}\left(v, a_{1}, a_{2}\right)\left(V_{p_{*}}(1)\right)\right)$ are disjoint for $-N \leq n \leq N$.

(IV) $p, q \in b_{p_{*}}\left(v, a_{1}, a_{2}\right)\left(V_{p_{*}}(\sqrt{ } 3 / 4)\right)$,

then there exists $g \in \mathscr{U}$ such that

(i ) $g^{2 N}\left(f^{-N}(q)\right)=f^{N}(p)$

(ii) $\sup g f^{-1} \subset \bigcup_{n=-N+1}^{N} f^{n}\left(b_{p_{*}}\left(v, a_{1}, a_{2}\right)\left(V_{p_{*}}(1)\right)\right)$.

When we apply the closing lemma in the succeeding sections, we require that the perturbation $g$ should preserve the $T f$-invariant subbundle $E^{1}, E^{2}$ and the norm of differentials restricted to them (for precise meaning, see (8.2) below). For that purpose, we must extend the splitting $T M \mid \Lambda$ $=E^{1} \oplus E^{2}$ to a neighborhood of $\Lambda$ beforehand. Since both $M$ and the 1-dimensional Grassmann bundle over $M$ (whose fiber over $x \in M$ is the Grassmann manifold of all 1-dimensional subspaces of $T_{x} M$ ) are $A N R$, we can extend $E^{i}$ continuously to a neighborhood $U$ of $\Lambda$ (see (4.4) Lemma in [2] for the proof). We still call them $E^{i}$. Although $E^{1}$ and $E^{2}$ are not necessarily $T$-invariant outside $\Lambda$, by restricting $U$ to a neighborhood sufficiently near $\Lambda$, we can assume that $T M \mid U=E^{1} \oplus E^{2}$.

The following is the version of the closing lemma which we shall use afterwards.

(8.2) Lemma. For any $C^{1}$-neighborhood $\mathscr{U}$ of $f$, there exist $r>0$ and $N \in Z_{+}$with the following property. Let $p_{*} \in \Lambda$ and $p, q \in M$. If $v \in T_{p_{*}} M$ and positive numbers $a_{1}, a_{2}$ satisfy

( I ) $|v|+a_{1}+a_{2} \leq r$

(II) $(2 \sqrt{2})^{-1} \leq a_{1} / a_{2} \leq 2 \sqrt{2}$

(III) $f^{n}\left(b_{p_{*}}\left(v, a_{1}, a_{2}\right)\left(V_{p_{*}}(1)\right)\right)$ are disjoint for $-N+1 \leq n \leq N$

(IV) $p, q \in b_{p_{*}}\left(v, a_{1}, a_{2}\right)\left(V_{p_{*}}(\sqrt{3 / 4})\right)$,

then there exists $g \in \mathscr{U}$ such that

(i ) $g^{2 N}\left(f^{-N}(q)\right)=f^{N}(p)$

(ii) $\operatorname{sup~} g f^{-1} \subset \bigcup_{n=-N+1}^{N} f^{n}\left(b_{p_{*}}\left(v, a_{1}, a_{\S}\right)\left(V_{p_{*}}(1)\right)\right)$

(iii) $\operatorname{Tg}\left(E_{g^{n}(f-N(q))}^{i}\right)=E_{g^{n+1}(f-N(q))}^{i}$ 
for all $0 \leq n \leq 2 N-1$ and $i=1,2$

$$
\left\|T g\left|E_{g^{n}(f-N(q))}^{i}\|=\| T f\right| E_{f^{n-N}(q)}^{i}\right\|
$$

for all $0 \leq n \leq N-1$ and $i=1,2$

$$
\left\|T g\left|E_{g^{n}(f-N(q))}^{i}\|=\| T f\right| E_{f^{n-N}(p)}^{i}\right\|
$$

for all $N \leq n \leq 2 N-1$ and $i=1,2$.

Proof. The basic idea of the proof is simple. We only have to perturb the differentials of $g$ in (8.1) around the points $\left\{g^{n}\left(f^{-N}(q)\right)\right\}_{0 \leq n \leq 2 N}$ by using (2.9).

Take $\varepsilon^{\prime}>0$ so that $\mathscr{U}\left(\varepsilon^{\prime}, f\right) \subset \mathscr{U} \cap \mathscr{U}_{2}$, where $\mathscr{U}_{2}$ is given in (2.9). Let $r>0$ and $N \in Z_{+}$be given in (8.1) with $\mathscr{U}\left(\varepsilon^{\prime} / 4, f\right)$ as a $C^{1}$-neighborhood of $f$. Let $p, q \in M, v \in T_{p_{*}} M$ and $a_{1}>0, a_{2}>0$, with the properties (I) (IV). Then from (8.1), there exists $g \in \mathscr{U}\left(\varepsilon^{\prime} / 4, f\right)$ satisfying (i) and (ii). Take $r>0$ so small that for all $-N \leq n \leq N, f^{n}\left(b_{p_{*}}\left(v, a_{1}, a_{2}\right)\left(V_{p_{*}}(1)\right)\right)$ are contained in $U$ on which extended $E^{1}$ and $E^{2}$ are defined. Define linear maps

$$
G_{j}^{q}: T_{g^{N+j(f-N(q))}} M \longrightarrow T_{f^{j}(q)} M \quad \text { for }-N \leq j \leq 0
$$

and

$$
G_{j}^{p}: T_{g^{N+j(f-N(q))}} M \longrightarrow T_{f^{j(p)}} M \quad \text { for } 0 \leq j \leq N
$$

so that they preserve $E^{1}, E^{2}$ and are isometry on them. Note that $G_{-N}^{q}$ and $G_{N}^{p}$ are identity maps on $T_{f^{-N}(q)} M$ and $T_{f^{N}(p)} M$ respectively.

Now we shall apply (2.9) to $g$ with $\left\{g^{n}\left(f^{-N}(q)\right)\right\}_{0 \leq n \leq 2 N-1}$ as a finite set. As linear maps, we take

$$
A_{j}=\left(G_{j+1}^{q}\right)^{-1}(T f)_{f^{j}(q)} G_{j}^{q}\left(T g^{-1}\right)_{g^{N+j+1(f-N(q))}} \quad \text { for }-N \leq j \leq-1
$$

and

$$
A_{j}=\left(G_{j+1}^{p}\right)^{-1}(T f)_{f^{j}(p)} G_{j}^{p}\left(T g^{-1}\right)_{g^{N+j+1}(f-N(q))} \quad \text { for } 0 \leq j \leq N-1 .
$$

By taking $r>0$ smaller, the distance from $f^{j}(q)$, resp. $f^{j}(p)$, to $g^{N+j}\left(f^{-N}(q)\right)$ for $-N \leq j \leq 0$, resp. $0 \leq j \leq N$, can be small enough to satisfy $\left\|A_{j}-\mathrm{id}\right\|<\delta^{\prime}$ for all $-N \leq j \leq N-1$, where $\delta^{\prime}>0$ is given in (2.9) with $\varepsilon=\varepsilon^{\prime} / 4$. Then from (2.9), we can find $g^{\prime}$ such that

(i) $d_{1}\left(g, g^{\prime}\right)<\varepsilon^{\prime} / 4$

(ii) $T g^{\prime}=A_{f} \circ T g$ on $T_{g^{N+j_{(f-N(q))}}} M$ for all $-N \leq j \leq N-1$. 
Moreover, the support of this perturbations can be arbitrarily small. This proves the lemma.

To simplify notations in the succeeding sections, we introduce the following definition.

(8.3) Definition. Let $p_{*} \in A, N \in Z_{+}$and $r>0$. Let $\left(p, q, v, a_{1}, a_{2}\right)$ be $\left(p_{*}, r\right)$-connectable, where $p, q \in M, v \in T_{p_{*}} M$ and $a_{1}>0, a_{2}>0$. Assume that $f^{n}\left(b_{p_{*}}\left(v, a_{1}, a_{2}\right)\left(V_{p_{*}}(1)\right)\right)$ are disjoint for $-N \leq n \leq N$ and contained in a neighborhood $U$ of $\Lambda$ on which extended $E^{1}$ and $E^{2}$ are defined. $C^{1}$ diffeomorphism $g$ is $\left(p_{*}, N, p, q, v, a_{1}, a_{2}\right.$ )-connector if and only if $g$ satisfies (i) (iv) in (8.2).

With this definition, we can formulate (8.2) as follows.

(8.4) Lemma. For any $C^{1}$-neighborhood $\mathscr{U}$ of $f$, there exist $r>0$ and $N \in Z_{+}$with the following property; let $p_{*} \in \Lambda, p, q \in M, v \in T_{p_{*}} M$ and $a_{1}>$ $0, a_{2}>0$. If $\left(p, q, v, a_{1}, a_{2}\right)$ is $\left(p_{*}, r\right)$-connectable and $f^{n}\left(b_{p_{*}}\left(v, a_{1}, a_{2}\right)\left(V_{p_{*}}(1)\right)\right)$ are disjoint for $-N+1 \leq n \leq N$, then there exists a $\left(p_{*}, N, p, q, v, a_{1}, a_{2}\right)$ connector $g$ in $\mathscr{U}$.

Remark that the definition of $\left(p_{*}, N, p, q, v, a_{1}, a_{2}\right)$-connector depends on the order of $p$ and $q$, so generally $\left(p_{*}, N, p, q, v, a_{1}, a_{2}\right)$-connector and $\left(p_{*}, N, q, p, v, a_{1}, a_{2}\right)$-connector are different. This difference plays a remarkable role in the proof of our main lemma. Roughly speaking, if $q=f^{n}(p)$ for some positive integer $n,\left(p_{*}, N, p, q, v, a_{1}, a_{2}\right)$-connector closes up the orbit $\left\{f^{k}(p)\right\}_{0 \leq k \leq n}$ and $\left(p_{*}, N, q, p, v, a_{1}, a_{2}\right)$-connector cuts off it.

\section{§9. Existence of a saddle}

In this section, we shall give the following technical lemma which asserts that a sufficiently near recurrence with exponential expansion of the norm of $T f$ on $E^{1}$ and contraction on $E^{2}$ implies the existence of a saddle.

(9.1) Lemma. There exist $N_{1} \in Z_{+}, r_{6}>0$ and $r_{7}>0$ such that if $p \in \Lambda$ and $m \in Z_{+}$satisfy

(i) $m \geq N_{1}$

(ii) $\left\|T f^{m} \mid E_{p}^{1}\right\| \geq\left(\lambda^{-9 / 10}\right)^{m}$

$\left\|T f^{m} \mid E_{p}^{2}\right\| \leq\left(\lambda^{9 / 10}\right)^{m}$

(iii) $\quad d\left(p, f^{m}(p)\right) \leq r_{6}$

(iv) Putting $(x, y)=\psi_{p}\left(f^{m}(p)\right)$ and $r=\max \{|x|,|y|\}, \quad B_{p}(4 r)$ is a $\left(p, m, r_{7}\right)$-tracing box, 
then, there is a saddle in $B_{p}(4 r)$. Moreover, the period of this saddle is $a$ divisor of $m$.

This kind of argument is used sometimes in the theory of hyperbolic sets, but in our case, the condition on the norm of differentials of $f^{k}$ at $p$ are not given for $0<k<m$. Instead, we have a condition on the size of the tracing box $B_{p}(4 r)$. While the basic idea of the proof is the same as in the case of hyperbolic sets, the precise estimation is rather messy.

Firstly, for a vector $v=\left[\begin{array}{l}v_{1} \\ v_{2}\end{array}\right] \in \boldsymbol{R}^{2}$ with $v_{1} \neq 0$, we define

$$
\text { slope }(v)=s(v)=\left|v_{2}\right| /\left|v_{1}\right| \text {. }
$$

For $A=\left[\begin{array}{ll}\alpha & \beta \\ \gamma & \delta\end{array}\right] \in G L(2, R)$ with $\alpha \neq 0, \delta \neq 0$, define

$$
h(A)=|\delta| /|\alpha| .
$$

Now we consider for $A$ the following condition,

$$
|\beta|,|\gamma| \leq \varepsilon, \quad a \leq|\alpha|,|\delta| \quad \text { for positive constants } \varepsilon, a .
$$

Then, we have easily,

(9.2) Lemma. If $A$ satisfies (1) and $1 \geq \varepsilon a^{-1} s(v)$, then

$$
s(A v) \leq h(A)\left(1-\varepsilon a^{-1} s(v)\right)^{-1}\left(\varepsilon a^{-1}+s(v)\right) .
$$

Next, let us consider a sequence $\left\{A_{i}=\left[\begin{array}{ll}\alpha_{i} & \beta_{i} \\ \gamma_{i} & \delta_{i}\end{array}\right]\right\}_{i \in Z_{+}}$satisfying (1) and furthermore

$$
\prod_{n=i}^{j} h\left(A_{n}\right) \leq c\left(\lambda^{9 / 10}\right)^{j-i+1} \quad \text { for all } i, j \in Z_{+} \text {with } i \leq j .
$$

We shall estimate the slope of $A_{n} \cdots A_{1} v$. For the sake of simplicity, we put

$$
\rho=\left(1-\lambda^{8 / 10}\right)^{-1}, \quad \mu=\lambda^{-1 / 10}, \quad \tau=\varepsilon a^{-1} .
$$

(9.3) Lemma. Assume that $s(v) \leq 8 \rho c \tau$ and let $\varepsilon>0$ be so small that $\left(1-c \rho(1+8 c) \tau^{2}\right)^{-1} \leq \mu$. Then we have

$$
\begin{array}{r}
s\left(A_{n} \cdots A_{1} v\right) \leq\left\{\sum_{i=1}^{n}\left(\prod_{j=i}^{n} \mu h\left(A_{j}\right)\right)+8 \rho c \prod_{j=1}^{n} \mu h\left(A_{j}\right)\right\} \tau \\
\text { for all } n \in Z_{+} .
\end{array}
$$

Proof. We shall prove the lemma by induction.

For $n=1$, by using $s(v) \leq 8 \rho c \tau$ and (9.2), we get 


$$
\begin{aligned}
s\left(A_{1} v\right) & \leq h\left(A_{1}\right)(1-\tau 8 \rho c \tau)^{-1}(\tau+8 \rho c \tau) \\
& \leq h\left(A_{1}\right) \mu(1+8 \rho c) \tau,
\end{aligned}
$$

because, $\left(1-\tau^{2} 8 \rho c\right)^{-1} \leq\left(1-c \rho(1+8 c) \tau^{2}\right)^{-1} \leq \mu$.

Suppose the lemma is true for $n-1$. Then from (2), we have

$$
\begin{aligned}
s\left(A_{n-1} \cdots A_{1} v\right) & \leq\left\{\sum_{i=1}^{n-1}\left(\prod_{j=i}^{n-1} \mu h\left(A_{j}\right)\right)+8 \rho c \prod_{j=1}^{n-1} \mu h\left(A_{j}\right)\right\} \tau \\
& \leq\left\{\sum_{i=1}^{n-1} \mu^{n-i} c\left(\lambda^{9 / 10}\right)^{n-i}+8 \rho c \mu^{n-1} c\left(\lambda^{9 / 10}\right)^{n-1}\right\} \tau \\
& \leq\left\{\sum_{i=1}^{\infty}\left(\lambda^{8 / 10}\right)^{i}+8 \rho c\left(\lambda^{8 / 10}\right)^{n-1}\right\} c \tau \\
& \leq\left\{1+8 c\left(\lambda^{8 / 10}\right)^{n-1}\right\} \rho c \tau .
\end{aligned}
$$

Namely,

$$
s\left(A_{n-1} \cdots A_{1} v\right) \leq\left\{1+8 c\left(\lambda^{8 / 10}\right)^{n-1}\right\} \rho c \tau .
$$

This implies that

$$
\left(1-\tau s\left(A_{n-1} \cdots A_{1} v\right)\right)^{-1} \leq\left(1-\tau^{2}(1+8 c) \rho c\right)^{-1} \leq \mu,
$$

and

$$
\begin{aligned}
\tau s\left(A_{n-1} \cdots A_{1} v\right) & \leq \tau^{2}(1+8 c) \rho c \\
& <1-\mu^{-1}<1 .
\end{aligned}
$$

so, by (9.2), we get

$$
s\left(A_{n} \cdots A_{1} v\right) \leq h\left(A_{n}\right) \mu \tau\left\{1+\sum_{i=1}^{n-1}\left(\prod_{\substack{n-1 \\ j=i}}^{n-1} \mu h\left(A_{j}\right)\right)+8 \rho c \prod_{j=1}^{n-1} \mu h\left(A_{j}\right)\right\}
$$

which implies (3) for $n$.

By the same calculation proving the above (4), we have

(9.4) LEMma. Under the same hypothesis as (9.3),

$$
s\left(A_{n} \cdots A_{1} v\right) \leq\left\{1+8 c\left(\lambda^{8 / 10}\right)^{n}\right\} \rho c \tau \quad \text { for all } n \in Z_{+} .
$$

Proof of (9.1). For $q \in \Lambda$ and $x \in B\left(r_{7}\right)$, we put

$$
T_{x} f_{q}=\left[\begin{array}{ll}
\alpha_{q} & \beta_{q} \\
\gamma_{q} & \delta_{q}
\end{array}\right] \text { and } T_{0} f_{q}=\left[\begin{array}{cc}
\alpha_{q}^{0} & 0 \\
0 & \delta_{q}^{0}
\end{array}\right]
$$

where $f_{q}=\psi_{f(q)} \circ f \circ \psi_{q}^{-1}$.

By taking $r_{7}$ small, we can assume that

(5) (i ) there is a constant $a>0$ such that

$$
a \leq\left|\alpha_{q}\right|,\left|\delta_{q}\right| \quad \text { for any } q \in \Lambda
$$

(ii) there is $\varepsilon>0$ such that $\left|\beta_{q}\right|,\left|\gamma_{q}\right| \leq \varepsilon$ for any $q \in \Lambda$ and

$$
\left(1-c \rho(1+8 c)\left(\varepsilon a^{-1}\right)^{2}\right)^{-1} \leq \mu
$$


(iii) $\lambda^{1 / 20} \leq\left|\alpha_{q}\right| /\left|\alpha_{q}^{0}\right| \leq \lambda^{-1 / 20}$

$\lambda^{1 / 20} \leq\left|\delta_{q}\right| /\left|\delta_{q}^{0}\right| \leq \lambda^{-1 / 20} \quad$ for any $q \in \Lambda$.

We put $\tau=\varepsilon a^{-1}$ as before, and $\psi_{p}\left(f^{m}(p)\right)=(x, y), r=\max \{|x|,|y|\}$ as in the assumption of the lemma.

Put $q=f^{m}(p)$ and let $z$ be an arbitraly point in $B(4 r)$. Take $v \in T_{z} R^{2}$ such that $s(v) \leq 8 \rho c \tau$. Let us consider $T_{z}\left(\psi_{p} \circ f^{m} \circ \psi_{p}^{-1}\right)(v)$. To simplify notation, put $f_{p}^{n}=\psi_{f n(p)} \circ f^{n} \circ \psi_{p}^{-1}, z_{n}=f_{p}^{n}(z)$ and $p_{n}=f^{n}(p)$ for $0 \leq n \leq m$.

Noting that $T_{z}\left(\psi_{p} \circ f^{m} \circ \psi_{p}^{-1}\right)=T_{z_{m}}\left(\psi_{p} \psi_{q}^{-1}\right) \circ T_{z} f_{p}^{m}$, first, we estimate the slope and the norm of $T_{z} f_{p}^{m}(v)$.

From (5) (i) (iii), (9.1) (iv) and (2.3) (ii), a sequence $\left\{T_{z_{n}} f_{p_{n}}\right\}_{n=0, \cdots, m-1}$ satisfies the hypothesis of (9.3). Therefore, if we take $m$ large enough so that $8 c\left(\lambda^{8 / 10}\right)^{m} \leq 1$, then by $(9.4), s\left(T_{z} f_{p}^{m}(v)\right) \leq 2 \rho c \tau$. If $d\left(p, f^{m}(p)\right)$ is sufficiently small (i.e. we take $r_{6}$ small enough), then by (5.4) (iv), we get $s\left(T_{z}\left(\psi_{p} \circ f^{m} \circ \psi_{p}^{-1}\right)(v)\right) \leq 4 \rho c \tau$.

Thus, $T_{z}\left(\psi_{p} \circ f^{m} \circ \psi_{p}^{-1}\right)$ preserves the sector

$$
S_{4 \rho c \tau}=\left\{v \in T_{x} R^{2}: s(v) \leq 4 \rho c \tau, x \in B\left(r_{7}\right)\right\}
$$

for $z \in B(4 r)$.

Next, we investigate the norm of $T_{z}\left(\psi_{p} \circ f^{m} \circ \psi_{p}^{-1}\right)(v)$. Assume that $v=\left[\begin{array}{l}v_{1} \\ v_{2}\end{array}\right]$ and $\left|v_{1}\right|=1$. Generally, if a matrix $A=\left[\begin{array}{ll}\alpha & \beta \\ \gamma & \delta\end{array}\right]$ satisfy the assumption of (9.2), then

$$
\begin{aligned}
\left|\pi_{1} A v\right| & =\left|\alpha v_{1}+\beta v_{2}\right| \geq|\alpha|\left|v_{1}\right|-|\beta|\left|v_{2}\right| \\
& \geq|\alpha|\left|v_{1}\right|\left(1-\varepsilon a^{-1} s(v)\right),
\end{aligned}
$$

where $\pi_{1}$ denotes the canonical projection to the first coordinate. From this (6), (9.4), (5) (iii) and (9.1) (ii), we have

$$
\begin{aligned}
\left|\pi_{1} T_{z} f_{p}^{m}(v)\right| \geq\left|\frac{\partial f_{p_{m-1}}^{1}}{\partial x_{1}}\left(z_{m-1}\right)\right| \cdot\left|\pi_{1} T_{z} f_{p}^{m-1}(v)\right|\left(1-\tau s\left(T_{z} f_{p}^{m-1}(v)\right)\right) \\
\geq\left|\frac{\partial f_{p_{m-1}}^{1}}{\partial x_{1}}\left(z_{m-1}\right)\right| \cdot\left|\pi_{1} T_{z} f_{p}^{m-1}(v)\right|\left(1-\tau^{2}(1+8 c) \rho c\right) \\
\geq\left|\frac{\partial f_{p_{m-1}}^{1}}{\partial x_{1}}\left(z_{m-1}\right)\right| \cdot\left|\pi_{1} T_{z} f_{p}^{m-1}(v)\right| \cdot \mu^{-1} \\
\\
\geq \\
\geq\left\{\prod_{i=0}^{m-1}\left|\frac{\partial f_{p_{i}}^{1}}{\partial x_{1}}\left(z_{i}\right)\right|\right\} \cdot \lambda^{m / 10} \\
\geq\left(\lambda^{-8 / 10}\right)^{m} \lambda^{m / 10}=\left(\lambda^{-7 / 10}\right)^{m}
\end{aligned}
$$


Namely, if we take $m$ large enough to satisfy $\left(\lambda^{-7 / 10}\right)^{m} \geq 5$, then we have $\left|\pi_{1} T_{z} f_{p}^{m}(v)\right| \geq 5$. If we take $d\left(p, f^{m}(p)\right)$ sufficiently small (i.e. by lessening $\left.r_{6}\right)$, using (5.4) (iv), we have $\left|\pi_{1} T_{z}\left(\psi_{p} \circ f^{m} \circ \psi_{p}^{-1}\right)(v)\right| \geq 4$.

Applying the same argument to $f^{-1}$, we have that;

(i) for $z \in \psi_{p} f^{m}\left(B_{p}(4 \mathrm{r})\right)$ and $v=\left[\begin{array}{l}v_{1} \\ v_{2}\end{array}\right] \in T_{z} R^{2}$ with $\left|v_{2}\right|=1$ and $\tilde{s}(v)=$ $\left|v_{1}\right| /\left|v_{2}\right| \leq 8 \rho c \tau, \psi_{p} \circ f^{-m} \circ \psi_{p}^{-1}$ preserves the sector

$$
\tilde{S}_{4 \rho c \tau}=\left\{v \in T_{x} R^{2}: \tilde{s}(v) \leq 4 \rho c \tau, x \in B\left(r_{7}\right)\right\}
$$

on $\psi_{p} f^{m}\left(B_{p}(4 r)\right)$.

(ii) $\left|\pi_{2} T_{z}\left(\psi_{p} \circ f^{-m} \circ \psi_{p}^{-1}\right)(v)\right| \geq 4$, where $\pi_{2}$ denotes the canonical projection to the second coordinate.

Thus, we know that $\psi_{p} \circ f^{m} \circ \psi_{p}^{-1}$ expands $B(4 r)$ with respect to the first coordinate and contracts with respect to the second coordinate. Then, by using the above properties, it can be seen that $\bigcap_{n \in Z} f^{m n}\left(B_{p}(4 r)\right)$ consists of only one point which must be a saddle.

\section{$\S 10$. Disjointness of tracing boxes}

The purpose of this section is to prove the following (10.2) Lemma which gives a precise estimate about the length of the positive orbit on which the iterated box neighborhoods are pairwise disjoint. This (10.2) plays two important roles in the arguments in Sections 12 and 15 . One is to guarantee the disjointness of the supports of perturbations when we apply the closing lemma, and another is to guarantee that the length of suborbits are not smaller than a given number. Refer to (15.1) and (15.2) for details.

We need the following constants to formulate (10.2).

(10.1) Constants. $N_{1} \in Z_{+}$is given in (9.1). $C^{1}$ neighborhood $\mathscr{U}_{1}$ of $f$ is given in (2.3) (i). Let $C^{1}$ neighborhood $\mathscr{U}_{3}$ of $f$ be given in (3.1) with $\varepsilon=(1 / 4) d\left(\Lambda_{1}, \Lambda_{0} \cup \Lambda_{2}\right)$. Let $r_{0}>0$ and $N_{0} \in Z_{+}$be given in (8.2) with $\mathscr{U}=$ $\mathscr{U}\left(\varepsilon_{0}, f\right)$, where $\varepsilon_{0}>0$ is chosen so that $\mathscr{U}\left(\varepsilon_{0}, f\right) \subset \mathscr{U}_{1} \cap \mathscr{U}_{3}$. Moreover, we assume that $N_{0}$ is so large that $c \lambda^{N_{0}} \leq 1$ and $r_{0}$ is small enough to satisfy $B_{p}\left(r_{0}\right) \subset U_{p}\left((1 / 8) d\left(\Lambda_{1}, \Lambda_{0} \cup \Lambda_{2}\right)\right)$ for any $p \in \Lambda$, where $U_{p}(r)$ denotes the $r$ neighborhood of $p$. Define $N_{2}=\max \left\{2 N_{1}, 4 N_{0}\right\}$ and $G=12\left(\log m_{0}^{-1}\right) /\left(\log \lambda^{-1}\right)$.

(10.2) Lemma. There exist $r_{8}>0$ and $N_{3} \in Z_{+}$with the following property. Let $p \in \Lambda$ and $0<r \leq r_{8}$. If integer $N \geq N_{3}$ satisfy $\log \eta(p, G N) \leq$ $(G N / 10)\left(\log \lambda^{-1}\right)$, then $B_{i}(r) \cap B_{j}(r)=\phi$ for any integers $0 \leq i<j \leq 2 N$, 
where $B_{i}(r)=B_{f^{i}(p)}(r \theta(p, i) / \eta(p, G N)(G N-i))$.

Main tools for the proof of (10.2) are (8.2) and (9.1). To apply them, we need that length of the suborbit of the positive orbit of $p \in A$ is not so small. So we must manage the case of short suborbits (i.e. $j-i$ is small) before proving (10.2), namely;

(10.3) Lemma. There exist $r_{8}^{\prime}>0$ and $N_{3}^{\prime} \in Z_{+}$with the following property. Let $p \in \Lambda$ and $0<r \leq r_{8}^{\prime}$. If integer $N \geq N_{3}^{\prime}$ satisfy $\log \eta(p, G N) \leq$ $(G N / 10)\left(\log \lambda^{-1}\right)$, then $B_{i}(r) \cap B_{j}(r)=\phi$ for any integers $0 \leq i<j \leq 3 N$ with $j-i \leq N_{2}$.

(10.4) Remark. Since $G \geq 12$ and $\theta(p, i) / \eta(p, G N) \leq 1$ for $i \leq 12 N$, we have $\theta(p, i) / \eta(p, G N)(G N-i) \leq 1$ for $i \leq 11 N$. Therefore $B_{i}(r)$ is welldefined if $i \leq 11 N$ and $r \leq r_{1} / 2$ (ref. (5.4)).

For the proof of (10.3), we need the following;

(10.5) Lemma. Let $n_{*} \in Z_{+}$be given. For any $\varepsilon>0$, there exists $\delta>0$ such that for $x \in M$ and a positive integer $k \leq n_{*}$ with $d\left(x, f^{k}(x)\right)<\delta$, there is a periodic point of $f$ in the $\varepsilon$-neighborhood of $x$. Moreover, the period of this periodic point is a divisor of $k$.

The proof is straightforward and left to the reader.

Proof of (10.3). Suppose that $B_{i}(r) \cap B_{j}(r) \neq \phi$ for some integers $0 \leq$ $i<j \leq 3 N$ with $j-i \leq N_{2}$. From (10.4), $\theta(p, i) / \eta(p, G N)(G N-i) \leq 1$ for $i \leq 11 N$. So, by taking $r \leq r_{3}$, we can apply (6.1) and have;

(i ) if $\theta(p, i) /(G N-i) \geq \theta(p, j) /(G N-j)$, then $f^{j}(p) \in B_{i}(3 r)$

(ii) if $\theta(p, i) /(G N-i) \leq \theta(p, j) /(G N-j)$, then $f^{i}(p) \in B_{j}(3 r)$.

Namely, we have

(1) there exist $q \in \Lambda$ and integers $0 \leq t \leq 3 N, 0<m \leq N_{2}$ such that $q, f^{m}(q) \in B_{t}(3 r)$.

In fact, take $m=j-i, q=f^{i}(p)$ and $t=i$ in case of (i), $t=j$ in case of (ii) accordingly.

Now we claim that;

(2) Let $K \geq 3$. If $K r \leq \min \left\{r_{3}, r_{4}\right\}$, then

$$
f^{m}\left(B_{t}(K r)\right) \subset B_{t}\left(10 m_{1}^{m} K r\right) \quad \text { where } m_{1}=\sup _{p \in A}\left\{\left\|T f\left|E_{p}^{1}\|,\| T f\right| E_{p}^{2}\right\|\right\} .
$$


In fact, from (6.4), we have $f^{m}\left(B_{t}(K r)\right) \subset B_{t+m}(c K r)$, and from (1), $f^{m}(q) \in B_{t}(K r) \cap B_{t+m}(c K r)$. Putting $\theta_{i}=\theta(p, i)$ and $\eta=\eta(p, G N)$, we get from (6.1) that;

$$
B_{t+m}(c K r) \subset B_{f^{t}(p)}\left(K r \theta_{t} / \eta(G N-t)+4 c K r \theta_{t+m} / \eta(G N-t-m)\right) .
$$

By taking $N$ with $9 N \geq 2 N_{2}$, we have $1 /(G N-t-m) \leq 2 /(G N-t)$ and

$$
\begin{gathered}
B_{f^{t}(p)}\left(K r \theta_{t} / \eta(G N-t)+4 c K r \theta_{t+m} / \eta(G N-t-m)\right) \\
\subset B_{f^{t}(p)}\left(K r \theta_{t}\left(1+8 c m_{1}^{m}\right) / \eta(G N-t)\right),
\end{gathered}
$$

because $\theta_{t+m}=\theta_{t} \cdot \theta\left(f^{t}(p), m\right), m \leq N_{2}$ and $\theta\left(f^{t}(p), m\right) \leq m_{1}^{m}$. Since $c \geq 1$ and $m_{1} \geq 1$, this proves (2).

From (1) and (2), we have that;

$$
q, f^{m}(q), f^{2 m}(q) \in B_{t}\left(10 \mathrm{~cm}_{1}^{m} 3 r\right) .
$$

Taking $r$ so that $3 r\left(10 \mathrm{~cm}_{1}^{N_{2}}\right)^{N_{1}-1} \leq \min \left\{r_{3}, r_{4}\right\}$ and applying (2) $N_{1}-1$ times, we get

$$
q, f^{m}(q), \cdots, f^{N_{1} m}(q) \in B_{t}\left(\left(10 \mathrm{~cm}_{1}^{m}\right)^{N_{1}-1} 3 r\right) .
$$

Choose $\varepsilon_{1}>0$ so that, for $x, y \in \Lambda$ with $d(x, y)<\varepsilon_{1}$ we have $\lambda^{1 / 10} \leq$ $\left\|T f\left|E_{x}^{i}\|/\| T f\right| E_{y}^{i}\right\| \leq \lambda^{-1 / 10}$ for $i=1,2$, and $\varepsilon_{2}>0$ so that, for $x, y \in M$ with $d(x, y)<\varepsilon_{2}, d\left(f^{n}(x), f^{n}(y)\right)<\varepsilon_{1}$ holds for all $0 \leq n \leq N_{2} N_{1}$.

Now let $\delta_{1}$ be a positive number given in (10.5) with $\varepsilon=\varepsilon_{2}$ and $n_{*}$ $=N_{2} N_{1}$.

Next, take $r$ so small that $B_{t}\left(K_{0} r\right)$ is contained in $\delta_{1} / 2$-neighborhood of $f^{t}(p)$, where we put $K_{0}=3\left(10 \mathrm{~cm}_{1}^{m}\right)^{N_{1}-1}$. Then, since $q, f^{m_{1}}(q) \in B_{t}\left(K_{0} r\right)$ and $N_{1} m \leq N_{2} N_{1}$, there exists a periodic point $z$ whose period is a divisor of $N_{1} m$ in $\varepsilon_{2}$-neighborhood of $q$ by (10.5). From the definition of $\varepsilon_{2}$,

$$
d\left(f^{n}(q), f^{n}(z)\right)<\varepsilon_{1} \quad \text { for all } 0 \leq n \leq N_{1} m .
$$

By taking $\varepsilon_{2}$ smaller than $(1 / 4) d\left(\Lambda_{1}, \Lambda_{0} \cup \Lambda_{2}\right)$, we know that $z$ is a saddle of $f$. Therefore, from (2.4),

$$
\begin{gathered}
\left\|T f^{\pi(z)} \mid E_{z}^{1}\right\| \geq \lambda^{-\pi(z)} \\
\left\|T f^{\pi(z)} \mid E_{z}^{2}\right\| \leq \lambda^{\pi(z)}
\end{gathered}
$$

where $\pi(z)$ denotes the period.

Since $N_{1} m$ is a multiple of $\pi(z)$, from (4), (5) and the definition of $\varepsilon_{1}$, we have 


$$
\begin{aligned}
\left\|T f^{N_{1} m} \mid E_{q}^{1}\right\| \geq\left(\lambda^{-9 / 10}\right)^{N_{1} m} \\
\left\|T f^{N_{1} m} \mid E_{q}^{2}\right\| \leq\left(\lambda^{9 / 10}\right)^{N_{1} m} .
\end{aligned}
$$

From (3), $q, f^{N_{1} m}(q) \in B_{t}\left(K_{0} r\right)$. Applying (6.1), we have $B_{t}\left(K_{0} r\right) \subset$ $B_{q}\left(4 K_{0} r \theta_{t} / \eta(G N-t)\right)$. Therefore,

$$
f^{N_{1} m}(q) \in B_{q}\left(4 K_{0} r \theta_{t} / \eta(G N-t)\right) .
$$

Since $f^{t}(p) \in B_{q}\left(4 K_{0} r \theta_{t} / \eta(G N-t)\right)$, from (6.1), we have

$$
B_{q}\left(16 K_{0} r \theta_{t} / \eta(G N-t)\right) \subset B_{t}\left(64 K_{0} r\right) .
$$

Take $r$ so small that $64 K_{0} r \leq r_{4}$. Then from (6.4),

$$
B_{t}\left(64 K_{0} r\right) \text { is a }\left(f^{t}(p), G N-t-1,64 c K_{0} r\right) \text {-tracing box . }
$$

Since $G \geq 12, t \leq 3 N$ and $m \leq N_{2}$, taking $N$ large, we can assume that $N_{1} m \leq G N-t-1$. So, from (6), (7), (8), $q$ and $m$ satisfy the hypothesis of (9.1). Applying (9.1), we know that there exists a saddle $z^{\prime}$ in $B_{q}\left(16 K_{0} r \theta_{t} l \eta(G N-i)\right)$ and the period of $z^{\prime}$ is a divisor of $N_{1} m$. So, from (8), we get;

(10) there exists a saddle $z^{\prime}$ whose period is a divisor of $N_{1} m$ in $B_{t}\left(64 K_{0} r\right)$.

One can easily see that if $N \geq N_{1} m$ then;

(11) there exists $k^{\prime} \in Z_{+}$such that

$$
5 N\left(\log m_{0}^{-1}\right) /\left(\log \lambda^{-1}\right) \leq k^{\prime} N_{1} m \leq G N-t-1 .
$$

Take $r$ so small that $64 c K_{0} r$-boxes are contained in $\varepsilon_{1}$-neighborhood for all points in $\Lambda$. Then from (9), (10), (11), we have that $d\left(f^{n}\left(z^{\prime}\right), f^{t+n}(p)\right)$ $<\varepsilon_{1}$ for all $0 \leq n \leq k^{\prime} N_{1} m$. From (2.4) and the definition of $\varepsilon_{1}$,

$$
\left\|T f^{k^{\prime} N_{1} m} \mid E_{f \iota(p)}^{1}\right\| \geq\left(\lambda^{-9 / 10}\right)^{k^{\prime} N_{1} m} .
$$

Now it remains only easy calculations to finish the proof. Using $\operatorname{dim} E^{1}=1$ and (11), (12), we have,

$$
\begin{aligned}
\log \left\|T f^{t+k^{\prime} N_{1} m} \mid E_{p}^{1}\right\| & =\log \left\|T f^{t}\left|E_{p}^{1}\|+\log \| T f^{k^{\prime} N_{1} m}\right| E_{f^{t}(p)}^{1}\right\| \\
& \geq-t\left(\log m_{0}^{-1}\right)+(9 / 10) k^{\prime} N_{1} m\left(\log \lambda^{-1}\right) \\
& \geq(9 N / 2)\left(\log m_{0}^{-1}\right)-3 N\left(\log m_{0}^{-1}\right) \\
& =(3 N / 2)\left(\log m_{0}^{-1}\right) .
\end{aligned}
$$

From (11), $t+k^{\prime} N_{1} m \leq G N-1$. Therefore, 
$\log \left\|T f^{t+k^{\prime} N_{1} m} \mid E_{p}^{1}\right\| \leq \log \eta(p, G N)$.

On the other hand, from the hypothesis of the lemma,

$$
\begin{aligned}
\log \eta(p, G N) & \leq(G N / 10)\left(\log \lambda^{-1}\right) \\
& =(6 N / 5)\left(\log m_{0}^{-1}\right),
\end{aligned}
$$

which is a contradiction. This completes the proof of (10.3).

Now we shall prove (10.2).

Assume that $B_{i}(r) \cap B_{j}(r) \neq \phi$ for $0 \leq i<j \leq 2 N$. By the same argument as in the proof of (10.3), we have

(13) there exist $q \in \Lambda$ and integers $0 \leq t \leq 2 N, 0 \leq m \leq 2 N$ such that $q, f^{m}(q) \in B_{t}(3 r)$.

Take $r$ so that $3 r \leq r_{5}$. Applying (7.4) with $\left\{f^{n}(q)\right\}_{0 \leq n \leq m}$ as a finite subset of $M$, we have from (13) that there exist $v \in T_{f^{t}(p)} M$, positive numbers $a_{1}, a_{2}$ and two points $f^{k_{1}}(q), f^{k_{2}}(q)$ with $0 \leq k_{1}<k_{2} \leq m$, such that $\left(f^{k_{1}}(q), f^{k_{2}}(q), v, a_{1}, a_{2}\right)$ is $\left(f^{t}(p), 90 r \theta_{t} / \eta(G N-t)\right)$-connectable and moreover,

$$
f^{n}(q) \notin b_{f^{t}(p)}\left(v, a_{1}, a_{2}\right)\left(V_{f^{t}(p)}(1)\right) \quad \text { for } 0 \leq n \leq m \text { with } n \neq k_{1}, k_{2} .
$$

We claim that;

$$
\left\{f^{n}\left(B_{t}(90 r)\right)\right\}_{-N_{0} \leq n \leq N_{0}} \quad \text { are disjoint . }
$$

In fact, suppose that $f^{n_{1}}\left(B_{t}(90 r)\right) \cap f^{n_{2}}\left(B_{t}(90 r)\right) \neq \phi$ for some $-N_{0} \leq n_{1}$ $<n_{2} \leq N_{0}$, namely,

$$
B_{t}(90 r) \cap f^{n_{2}-n_{1}}\left(B_{t}(90 r)\right) \neq \phi \quad \text { and } \quad 0 \leq n_{2}-n_{1} \leq 2 N_{0} .
$$

From (6.4), we have that

$$
f^{n_{2}-n_{1}}\left(B_{t}(90 r)\right) \subset B_{t+n_{2}-n_{1}}(90 r c) .
$$

Since $c \geq 1$,

$$
B_{t}(90 c r) \cap B_{t+n_{2}-n_{1}}(90 c r) \neq \phi .
$$

If we take $N \geq 2 N_{0}$, then $t+n_{2}-n_{1} \leq 2 N+2 N_{0} \leq 3 N$. By taking $r$ with $90 \mathrm{cr} \leq r_{8}^{\prime}$, (16) contradicts (10.3) and this proves (15).

With (15), we can apply the closing lemma to $\left(f^{k_{1}}(q), f^{k_{2}}(q), v, a_{1}, a_{2}\right)$. Take $r$ so that $90 r \leq r_{0}$. From (8.4) and (10.1), we have that there exists a $\left(f^{t}(p), N_{0}, f^{k_{1}}(q), f^{k_{2}}(q), v, a_{1}, a_{2}\right)$-connector $g$ in $\mathscr{U}\left(\varepsilon_{0}, f\right)$.

From (14), $f^{k_{2}-N_{0}}(q)$ is a periodic point of $g$ whose period is $k_{2}-k_{1}$. Put $q_{0}=f^{k_{2}-N_{0}}(q)$. From the definition of $r_{0}$ (ref. (10.1)), $q_{0}$ is a saddle of 
g. Since $g$ preserves $E^{1}$ and $E^{2}$, by using (2.3) (ii), we see that $E_{q_{0}}^{1}=$ $E_{q_{0}}^{u}(g)$ and $E_{q_{0}}^{2}=E_{q_{0}}^{s}(g)$. Therefore, from (2.4),

$$
\begin{aligned}
\left\|T g^{k_{2}-k_{1}} \mid E_{q_{0}}^{1}\right\| & \geq\left(\lambda^{-1}\right)^{k_{2}-k_{1}} \\
\left\|T g^{k_{2}-k_{1}} \mid E_{q_{0}}^{2}\right\| & \leq \lambda^{k_{2}-k_{1}} .
\end{aligned}
$$

So, from the properties of $g$ (ref. (8.3) and (8.2) (iv)), we get;

$$
\begin{aligned}
& \left\|T f^{k_{2}-k_{1}} \mid E_{f}^{1} k_{1(q)}\right\| \geq\left(\lambda^{-1}\right)^{k_{2}-k_{1}} \\
& \left\|T f^{k_{2}-k_{1}} \mid E_{f}^{1} k_{1(q)}\right\| \leq \lambda^{k_{2}-k_{1}} .
\end{aligned}
$$

Recall that $f^{k_{1}}(q), f^{k_{2}}(q) \in B_{t}(90 r)$. From (6.1), we have

$$
\begin{aligned}
& f^{k_{2}}(q) \in B_{t}(90 r) \subset B_{f^{k_{1}(q)}}\left(360 r \theta_{t} / \eta(G N-t)\right), \\
& B_{f^{k_{1}(q)}}\left(16 \cdot 90 r \theta_{t} / \eta(G N-t)\right) \subset B_{t}(64 \cdot 90 r) .
\end{aligned}
$$

By taking $r$ with $64.90 r \leq r_{4}$, we have from (6.4) that

$$
B_{t}(64 \cdot 90 r) \text { is a }\left(f^{t}(p), G N-t-1,64 \cdot 90 c r\right) \text {-tracing box . }
$$

By the same argument as in the proof of (15), it can be seen that $k_{2}-k_{1} \geq 2 N_{1}$. Since $k_{2}-k_{1} \leq 2 N$ and $G N-t \geq 10 N, G N-t-1>9 N$ $>k_{2}-k_{1}$. Thus, from (17), (18), (19) and (20), taking $r$ with $64.90 \mathrm{cr} \leq r_{7}$, we can apply (9.1) and have a periodic point $z$ in $B_{f^{k_{1}(q)}}\left(16 \cdot 90 \mathrm{r} \theta_{t} / \eta(G N-t)\right)$ whose period is a divisor of $k_{2}-k_{1}$. Therefore from (19), we get;

(21) there exists a saddle $z$ in $B_{t}(64.90 r)$ whose period is a divisor of $k_{2}-k_{1}$.

The rest of the proof is the same as that of (10.3).

\section{$\S 11$. Main lemma and Key lemma}

In this section, we state (11.2) Main lemma and by using it, prove (11.1) Key lemma which is literally the key to the proof of our theorem. The proof of (11.2) will be given in Section 15 .

(11.1) Key Lemma. There exists a constant $A_{0}>0$ such that if for $m \in Z_{+}$and $\ell>0$,

$$
\log \left\|T f^{m} \mid E_{p}^{1}\right\| \leq-\ell
$$

holds at some $p \in \Lambda$, then $m \geq A_{0} \cdot \ell^{4}$.

For the statement of our Main lemma, we put 


$$
G_{1}=\left(\log \lambda^{-1}\right) / 8\left(\log m_{0}^{-1}\right)
$$

and

$$
\nu_{0}(N)=\left[G_{1} N / 4 N_{0}\right] \quad \text { for an integer } N .
$$

In what follows, we use a terminology " $Z_{+}$-interval" which means a interval in $Z_{+}$, and denote it by $I=[u, v]$ for $u, v \in Z_{+}$with $u \leq v$, namely,

$$
I=[u, v]=\left\{n \in Z_{+}: u \leq n \leq v\right\} .
$$

We define length $(I)=v-u$.

(11.2) MaIN Lemma. There exists an integer $N_{*} \geq 8 N_{0} / G_{1}$ with the following property: Let $p \in \Lambda$. If for an integer $N \geq N_{*}$ and $m \in Z_{+}$,

$$
\log \left\|T f^{m} \mid E_{p}^{1}\right\| \leq\left(N^{10 / 9} / 2\right)(\log \lambda)
$$

holds, then there exist integers $0 \leq m^{\prime}<m, 0<\nu \leq \nu_{0}(N)$, and $\nu$ disjoint $Z_{+}$-intervals $\left\{I_{i}=\left[u_{i}, v_{i}\right]\right\}_{1 \leq i \leq \nu}$ in $\left[0, m-m^{\prime}\right]$ with the following properties;

(i ) $\left\|T f^{n} \mid E_{f^{\prime}(p)}^{1}\right\| \leq 1$ for all $0 \leq n \leq m-m^{\prime}$,

(ii) $\sum_{i=1}^{\nu}\left(v_{i}-u_{i}\right)>\nu_{0}(N) \cdot N$,

(iii) $\left\|T f^{v_{i}-u_{i}} \mid E_{f u_{i}+m^{\prime}(p)}^{1}\right\| \geq\left(\lambda^{-1}\right)^{v_{i}-u_{i}}$ for all $i=1, \cdots, \nu$.

(11.3) Remark. Since $G_{1} \leq 1 / 8, N_{*} \geq 64 N_{0} \geq 64$.

Now we prove (11.1) assuming (11.2). For that purpose, we consider the following proposition depending on two numbers $\theta \geq 1$ and $A>0$.

Prop. $[\theta, A]$. If for $m \in Z_{+}$and $\ell>0$,

$$
\log \left\|T f^{m} \mid E_{p}^{1}\right\| \leq-\ell \quad \text { holds at some } p \in \Lambda,
$$

then $m \geq A \cdot \ell^{\theta}$.

If Prop. $[\theta, A]$ is true for some $\theta \geq 4$ and for some constant $A>0$, then clearly we get (11.1). In what follows, we shall investigate for what values of $\theta$ and $A$, Prop. $[\theta, A]$ holds.

As the first step to see this, we give;

(11.4) Lemma. Prop. [1, $\left.\left(\log m_{0}^{-1}\right)^{-1}\right]$ holds.

Recalling that $m_{0}=\inf _{p \in \Lambda}\left\{\left\|T f\left|E_{p}^{1}\|\| T f,\right| E_{p}^{2}\right\|\right\}$, this lemma is obvious.

Now our strategy is to increase the value of $\theta$ by applying (11.2) inductively, namely;

(11.5) Lemma. Assume that Prop. $[\theta, A]$ is true and for $m \in Z_{+}$and $\ell>0$, we have; 
(i) $\quad \ell \geq\left(N_{*}^{10 / 9} / 2\right)\left(\log \lambda^{-1}\right)$

(ii) $\log \left\|T f^{m} \mid E_{p}^{1}\right\| \leq-\ell$ for some $p \in \Lambda$, then $m \geq k(\theta) \cdot A \cdot \ell^{9(1+\theta) / 10}$, where $k(\theta)=2^{-(\theta+11) / 10} \cdot\left(\log \lambda^{-1}\right)^{(\theta-9) / 10} \cdot G_{1} / N_{0}$.

Proof. We put

$$
N=\left[\left(2 \ell /\left(\log \lambda^{-1}\right)\right)^{9 / 10}\right]+1 .
$$

Then, from the assumption on $m, \ell$ and $N_{*}$, we have

$$
N \geq N_{*} \geq 8 N_{0} / G_{1} \text { and } \log \left\|T f^{m} \mid E_{p}^{1}\right\| \leq\left(N^{10 / 9} / 2\right)(\log \lambda) .
$$

Therefore we can apply (11.2) and have that; there are integers $0 \leq m^{\prime}$ $<m_{0}, 0<\nu \leq \nu_{0}(N)$ and $\nu$ disjoint $Z_{+}$-intervals $\left\{I_{i}\right\}_{1 \leq i \leq \nu}$ in $\left[0, m-m^{\prime}\right]$ with the properties (i) $\sim$ (iii) in (11.2). We put $I_{i}=\left[u_{i}, v_{i}\right]$. Without loss of generality, we can assume that $I_{1}<I_{2}<\cdots<I_{\nu}$ (i.e. $v_{i-1}<u_{i}$ for $2 \leq i \leq \nu$ ).

Define disjoint $Z_{+}$-intervals $\left\{\tilde{J}_{i}\right\}_{1 \leq i \leq \nu}$ in $\left[0, u_{\nu}\right]$ as follows.

$$
\tilde{J}_{1}=\left[0, u_{1}\right] \text { and } \quad \tilde{J}_{i}=\left[v_{i-1}, u_{i}\right] \quad \text { for } 2 \leq i \leq \nu .
$$

We put $q=f^{m^{\prime}}(p)$ and

$$
\begin{aligned}
& -\tilde{a}_{1}=\log \left\|T f^{u_{1}} \mid E_{q}^{1}\right\| \\
& -\tilde{a}_{i}=\log \left\|T f^{u_{i}-v_{i-1}} \mid E_{f^{v_{i-1}(q)}}^{1}\right\| \quad \text { for } 2 \leq i \leq \nu .
\end{aligned}
$$

From (11.2) (iii), (3) and $\operatorname{dim} E^{1}=1$, we have

$$
\begin{aligned}
\log \left\|T f^{v_{\nu}} \mid E_{q}^{1}\right\|= & \log \left\|T f^{u_{1}}\left|E_{q}^{1}\|+\log \| T f^{v_{1}-u_{1}}\right| E_{f}^{1} u_{1(q)}\right\| \\
& +\log \left\|T f^{u_{2}-v_{1}}\left|E_{f^{v_{1}(q)}}^{1}\|+\cdots+\log \| T f^{v_{\nu}-u_{\nu}}\right| E_{f}^{1} u_{\nu(q)}\right\| \\
\geq & -\tilde{a}_{1}+\log \left(\lambda^{-1}\right)^{v_{1}-u_{1}}+\left(-\tilde{a}_{2}\right)+\cdots+\left(-\tilde{a}_{\nu}\right)+\log \left(\lambda^{-1}\right)^{v_{\nu}-u_{\nu}} .
\end{aligned}
$$

On the other hand, from (11.2) (i),

$$
\log \left\|T f^{v_{\nu}} \mid E_{q}^{1}\right\| \leq 0 \text {. }
$$

So, we have

$$
\left(-\sum_{i=1}^{\nu} \tilde{a}_{i}\right)+\left(\log \lambda^{-1}\right) \sum_{i=1}^{\nu}\left(v_{i}-u_{i}\right) \leq 0 .
$$

We select intervals in $\left\{\tilde{J}_{i}\right\}_{1 \leq i \leq \nu}$ with the property that $\tilde{a}_{i}>0$ and denote them by $J_{1}<J_{2}<\cdots<J_{\nu^{\prime}}$. Put $J_{i}=\left[s_{i}, t_{i}\right]$ and

$$
-a_{i}=\log \left\|T f^{t_{i-s i}} \mid E_{f^{s i(q)}}^{1}\right\| \quad \text { for } 1 \leq i \leq \nu^{\prime} .
$$

Note that $a_{i}>0$ for all $1 \leq i \leq \nu^{\prime}$. Clearly

$$
\nu^{\prime} \leq \nu
$$




$$
\sum_{i=1}^{\nu} \tilde{a}_{i} \leq \sum_{i=1}^{\nu^{\prime}} a_{1} .
$$

From (11.2) (ii) and (2),

$$
\begin{aligned}
\sum_{i=1}^{v}\left(v_{i}-u_{i}\right) & >\nu_{0}(N) \cdot N=\left[G_{1} N / 4 N_{0}\right] \cdot N \\
& \geq\left(G_{1} / 4 N_{0}-1 / N\right) \cdot N^{2} \geq\left(G_{1} / 8 N_{0}\right) \cdot N^{2},
\end{aligned}
$$

namely;

$$
\sum_{i=1}^{\nu}\left(v_{i}-u_{i}\right)>\left(G_{1} / 8 N_{0}\right) \cdot N^{2} .
$$

From (4), (7) and (8),

$$
\sum_{i=1}^{\nu^{\prime}} a_{i} \geq\left(G_{1}\left(\log \lambda^{-1}\right) / 8 N_{0}\right) \cdot N^{2} .
$$

From hypothesis, we can apply Prop. $[\theta, A]$ to (5) and have

$$
t_{i}-s_{i}=\operatorname{length}\left(J_{i}\right) \geq A \cdot a_{i}^{\theta} \quad \text { for all } 1 \leq i \leq \nu^{\prime} .
$$

First, we consider the case of $\theta=1$. From (9) and (10) with $\theta=1$, one can easily see that

$$
\sum_{i=1}^{\nu^{\prime}} \text { length }\left(J_{i}\right) \geq\left(A G_{1}\left(\log \lambda^{-1}\right) / 8 N_{0}\right) \cdot N^{2} .
$$

Next, we assume that $\theta>1$. From (10),

$$
A^{-1} \cdot \sum_{i=1}^{\nu^{\prime}} \text { length }\left(J_{i}\right) \geq \sum_{i=1}^{\nu^{\prime}} a_{i}^{\theta} .
$$

By the Hölder's inequality, we have

$$
\left\{\sum_{i=1}^{\nu^{\prime}} a_{i}^{\theta}\right\}^{1 / \theta} \cdot\left(\nu^{\prime}\right)^{1-(1 / \theta)} \geq \sum_{i=1}^{\nu^{\prime}} a_{i} .
$$

From (6), $\nu^{\prime} \leq G_{1} N / 4 N_{0}$. Since $1-(1 / \theta)>0$,

$$
\left(\nu^{\prime}\right)^{1-(1 / \theta)} \leq\left(G_{1} N / 4 N_{0}\right)^{1-(1 / \theta)} .
$$

From (12), (13) and (14), we have

$$
\sum_{i=1}^{v^{\prime}} a_{i} \leq\left\{A^{-1} \cdot \sum_{i=1}^{\nu^{\prime}} \text { length }\left(J_{i}\right)\right\}^{1 / \theta} \cdot\left(G_{1} N / 4 N_{0}\right)^{1-(1 / \theta)} .
$$

From this (15) and (9),

$$
\begin{aligned}
& \left\{A^{-1} \cdot \sum_{l=1}^{v^{\prime}} \text { length }\left(J_{i}\right)\right\}^{1 / \theta} \cdot\left(G_{1} N / 4 N_{0}\right)^{1-(1 / \theta)} \\
& \quad \geq\left(G_{1}\left(\log \lambda^{-1}\right) / 8 N_{0}\right) \cdot N^{2} .
\end{aligned}
$$

By an easy calculation, we have

$$
\sum_{i=1}^{v^{\prime}} \text { length }\left(J_{i}\right) \geq\left(\left(\log \lambda^{-1}\right) / 2\right)^{\theta} \cdot\left(A G_{1} / 4 N_{0}\right) \cdot N^{1+\theta} .
$$

Although we proved (17) for $\theta>1$, if we put $\theta=1$ in (17), this coincides with (11). Namely, (17) is valid for $\theta \geq 1$. 
From (1), we have

$$
N \geq\left\{2 \ell /\left(\log \lambda^{-1}\right)\right\}^{9 / 10} .
$$

By substituting it in (17), we have

$$
\sum_{i=1}^{\nu^{\prime}} \text { length }\left(J_{i}\right) \geq 2^{-(\theta+11) / 10} \cdot\left(\log \lambda^{-1}\right)^{(\theta-9) / 10} \cdot\left(A G_{1} / N_{0}\right) \cdot \ell^{9(1+\theta) / 10} .
$$

Since $\left\{J_{i}\right\}$ are disjoint intervals in $\left[0, m-m^{\prime}\right]$,

$$
m>\sum_{i=1}^{\nu^{\prime}} \text { length }\left(J_{i}\right) \text {. }
$$

This completes the proof.

Now we remark that we can take away formally the hypothesis (i) in (11.5). In fact, put $\ell_{0}=N_{*}^{10 / 9}\left(\log \lambda^{-1}\right) / 2$, and we shall consider the case of $0<\ell<\ell_{0}$. Suppose that for $m \in Z_{+}$and $0<\ell<\ell_{0}$,

$$
\log \left\|T f^{m} \mid E_{p}^{1}\right\| \leq-\ell
$$

holds at some $p \in \Lambda$. Then $m \geq 1$, because $\ell>0$. Putting $k_{0}(\theta)=\ell_{0}^{-9(1+\theta) / 10}$, we get $k_{0}(\theta) \cdot \ell^{9(1+\theta) / 10}<1$, namely,

$$
m \geq k_{0}(\theta) \cdot \ell^{9(1+\theta) / 10} .
$$

Now define $k_{0}(\theta, A)=\min \left\{k(\theta) \cdot A, k_{0}(\theta)\right\}$. Then, from (11.5), we have;

(11.6) Lemma. If Prop. $[\theta, A]$ holds, then Prop. $\left[9(1+\theta) / 10, k_{0}(\theta, A)\right]$ also holds.

Recall that Prop. [1, $\left.\left(\log m_{0}^{-1}\right)^{-1}\right]$ is true by (11.4). Starting from this, by applying (11.6) repeatedly more than 5 times, we can get Prop. $\left[\theta, A_{0}\right]$ for $\theta>4$ and for some constant $A_{0}>0$. This implies (11.1) Key lemma.

Remark that applying (11.6) much more times, we can have Prop. [ $\theta, A]$ for $\theta$ arbitrarily close to 9 . But we don't need such large $\theta$.

\section{$\S 12$. Expansive intervals}

(12.1) Definition. Let $p \in \Lambda$ and $N \in Z_{+}$. A $Z_{+}$-interval $I=[u, v]$ is called a $(p, N)$-expansive interval if and only if $I$ satisfies

(i) length $(I)=v-u \geq N$

(ii) $\left\|T f^{v-u} \mid E_{f u(p)}^{1}\right\| \geq\left(\lambda^{-1 / 2}\right)^{v-u}$.

(12.2) Lemma. There exists $N_{4} \in Z_{+}$such that if

$$
\log \left\|T f^{m} \mid E_{p}^{1}\right\| \leq(N / 2)(\log \lambda) \quad \text { for } p \in \Lambda, m \in Z_{+}
$$

and integer $N \geq N_{4}$, then there exists a $(p, N)$-expansive interval in $[0, m]$. 
(12.3) Lemma. For any $p \in \Lambda$ and $N \in Z_{+}$, there exists $a(p, N)$ expansive interval.

The essence of the proof of our Theorem is in (12.2). (12.3) merely asserts the existence of the expansive intervals and doesn't claim where such intervals exist in $Z_{+}$. By a straightforward argument with rather rough estimates, one can prove (12.3) using (10.5) and (8.2), so, the proof is left to the reader. In the rest of this section, we shall prove (12.2).

Suppose for $p \in \Lambda$, and $m, N \in Z_{+}$, it holds that;

$$
\log \left\|T f^{m} \mid E_{p}^{1}\right\| \leq(N / 2)(\log \lambda) .
$$

Let $0 \leq k<m$ be the largest integer such that

$$
\log \left\|T f^{k} \mid E_{p}^{1}\right\| \geq(N / 4)(\log \lambda)+\left(\log m_{0}\right) .
$$

Then, we can see that;

$$
\begin{aligned}
& \log \left\|T f^{k} \mid E_{p}^{1}\right\|<(N / 4)(\log \lambda), \\
& \left\|T f^{n} \mid E_{f^{k}(p)}^{1}\right\| \leq 1 \quad \text { for all } 0 \leq n \leq m-k, \\
& \log \left\|T f^{m-k} \mid E_{f^{k}(p)}^{1}\right\| \leq(N / 4)(\log \lambda)-\left(\log m_{0}\right) .
\end{aligned}
$$

From (2), $\log \left\|T f^{k} \mid E_{p}^{1}\right\|<0$. Therefore, by applying (2.7) with $\rho=\exp \left(N^{-4 / 3}\right)$, we have $\nu=\left[k N^{-4 / 3} /\left(N^{-4 / 3}-\left(\log m_{0}\right)\right)\right]+1$ integers $0 \leq k_{1}<k_{2} \cdots<k_{\nu} \leq k$ such that

$$
\begin{aligned}
& \log \left\|T f^{n} \mid E_{f^{k_{i}(p)}}\right\| \leq n N^{-4 / 3} \\
& \qquad \text { for all } 0 \leq n \leq k-k_{i} \text { and } i=1,2, \cdots, \nu .
\end{aligned}
$$

From (3), we can easily see that

$$
\begin{aligned}
\log \left\|T f^{n} \mid E_{f k_{i(p)}}^{1}\right\| & \leq n N^{-4 / 3} \\
& \text { for all } 0 \leq n \leq m-k_{i} \text { and } i=1,2, \cdots, \nu .
\end{aligned}
$$

From (4),

$$
\log \left\|T f^{m-k} \mid E_{f^{k}(p)}^{1}\right\| \leq-\left\{(N / 4)\left(\log \lambda^{-1}\right)-\left(\log m_{0}^{-1}\right)\right\} .
$$

Taking $N$ so large that $(N / 4)\left(\log \lambda^{-1}\right)>\log m_{0}^{-1}$, and applying (11.1), we have

$$
\begin{aligned}
m-k & \geq A_{0}\left\{(N / 4)\left(\log \lambda^{-1}\right)-\left(\log m_{0}^{-1}\right)\right\}^{4} \\
& =A_{0}\left\{(1 / 4)\left(\log \lambda^{-1}\right)-(1 / N)\left(\log m_{0}^{-1}\right)\right\}^{4} \cdot N^{4} .
\end{aligned}
$$

If we choose $N$ so that 


$$
(1 / 4)\left(\log \lambda^{-1}\right)-(1 / N)\left(\log m_{0}^{-1}\right) \geq(1 / 8)\left(\log \lambda^{-1}\right),
$$

then we have

$$
m-k \geq A_{1} N^{4}, \quad \text { where } A_{1}=A_{0}\left\{(1 / 8)\left(\log \lambda^{-1}\right)\right\}^{4} .
$$

Now we fix a constant $r>0$ such that

(8) $270 c r \leq r_{8}$ and $90 r<r_{0}, \quad$ where $r_{8}$ is given in (10.2).

We consider boxes $B_{f^{k_{1}(p)}}(r / G N)$ for each $f^{k_{i}}(p)$. From (5.4) (vi),

$$
\begin{aligned}
s\left(B_{f k_{i(p)}}(r / G N)\right) & \geq c_{1} s(B(r / G N)) \\
& =c_{1}(2 r / G N)^{2}, \quad \text { where } s(\cdot) \text { denotes the area } .
\end{aligned}
$$

Now let us estimate $\nu$. From (2),

$$
\log \left\|T f^{k} \mid E_{p}^{1}\right\| \leq-\left\{(N / 4)\left(\log \lambda^{-1}\right)\right\} .
$$

From (11.1), $k \geq A_{0}\left\{(N / 4)\left(\log \lambda^{-1}\right)\right\}^{4}$. Therefore,

$$
k \geq A_{1} N^{4} \text {. }
$$

From this (10),

$$
k N^{-4 / 3} /\left(N^{-4 / 3}-\log m_{0}\right) \geq A_{1} N^{4} N^{-4 / 3} /\left(N^{-4 / 3}+\log m_{0}^{-1}\right) .
$$

Taking $N$ with $N^{-4 / 3} \leq \log m_{0}^{-1}$, we have

$$
\begin{aligned}
\nu & >k N^{-4 / 3} /\left(N^{-4 / 3}-\log m_{0}\right) \\
& \geq\left\{A_{1} / 2\left(\log m_{0}^{-1}\right)\right\} \cdot N^{8 / 3} .
\end{aligned}
$$

Namely, putting $A_{2}=A_{1} / 2\left(\log m_{0}^{-1}\right)$, we get;

$$
\nu>A_{2} N^{8 / 3} \text {. }
$$

We consider the sum of the area of $B_{f k_{i(p)}}(r / G N)(i=1, \cdots, \nu)$. By (9) and (11),

$$
\begin{aligned}
\sum_{i=1}^{\nu} s\left(B_{f k_{i}(p)}(r / G N)\right) & \geq \sum_{i=1}^{\nu} c_{1}(2 r / G N)^{2} \\
& >A_{2} c_{1}(2 r / G)^{2} \cdot N^{2 / 3},
\end{aligned}
$$

which exceeds the surface area of $M$ by taking sufficiently large $N$. Therefore,

(12) there exist at least two points $f^{k_{i}}(p), f^{k_{j}}(p)$ such that

$$
B_{f k_{i(p)}}(r / G N) \cap B_{f^{k(p)}}(r / G N) \neq \phi .
$$


Assume that $k_{i}<k_{j}$ and put $f^{k_{i}}(p)=p_{0}, f^{k_{j}}(p)=q_{0}$ and $k_{j}-k_{i}=k_{0}$. By taking $N$ with $r / G N \leq r_{3}$, where $r_{3}$ is from (6.1), and applying (6.1), we have;

$$
q_{0} \in B_{p_{0}}(3 r / G N) .
$$

Then applying (7.4) with $\left\{f^{n}\left(p_{0}\right)\right\}_{0 \leq n \leq k_{0}}$ as a finite set, we have from (13) that;

(14) there exist $v \in T_{p_{0}} M$, positive numbers $a_{1}, a_{2}$ and two points $f^{n_{1}}\left(p_{0}\right)$, $f^{n_{2}}\left(p_{0}\right)$ with $0 \leq n_{1}<n_{2} \leq k_{0}$, such that $\left(f^{n_{1}}\left(p_{0}\right), f^{n_{2}}\left(p_{0}\right), v, a_{1}, a_{2}\right)$ is $\left(p_{0}, 90 r / G N\right)$-connectable and $f^{n}\left(p_{0}\right) \notin b_{p_{0}}\left(v, a_{1}, a_{2}\right)\left(V_{p_{0}}(1)\right)$ for all $0 \leq n$ $\leq k_{0}$ with $n \neq n_{1}, n_{2}$.

We want to close up this suborbit from $f^{n_{1}}\left(p_{0}\right)$ to $f^{n_{2}}\left(p_{0}\right)$ using closing lemma, but we need some preparations beforehand.

By taking $N$ so large that $A_{1} N^{4} \geq 2 G N$ and $2 G N^{-1 / 3} \leq 1$, we have from (6), (7);

$$
\log \left\|T f^{n} \mid E_{p_{0}}^{1}\right\| \leq n N^{-4 / 3} \leq 1 \quad \text { for all } 0 \leq n \leq 2 G N .
$$

Thus, we have;

$$
\log \eta\left(p_{0}, G N\right) \leq 1 \text {. }
$$

Next, take $N$ with $N \geq N_{3}$ and $(G N / 10)\left(\log \lambda^{-1}\right) \geq 1$, then by (8) and (10.2), we have;

$$
B_{i}(270 \mathrm{cr}) \cap B_{j}(270 \mathrm{cr})=\phi \quad \text { for any integer } 0 \leq i<j \leq 2 N,
$$

where

$$
B_{i}(270 c r)=B_{f^{i}\left(p_{0}\right)}\left(270 c r \theta\left(p_{0}, i\right) / \eta\left(p_{0}, G N\right)(G N-i)\right) .
$$

We claim that;

$$
f^{n}\left(B_{p_{0}}(90 r / G N)\right) \quad \text { are disjoint for }-N_{0} \leq n \leq N_{0} .
$$

In fact, assume that

$$
f^{n}\left(B_{p_{0}}(90 r / G N)\right) \cap f^{n^{\prime}}\left(B_{p_{0}}(90 r / G N)\right) \neq \phi,
$$

for some $-N_{0} \leq n<n^{\prime} \leq N_{0}$, namely,

$$
B_{p_{0}}(90 r / G N) \cap f^{n^{\prime}-n}\left(B_{p_{0}}(90 r / G N)\right) \neq \phi .
$$

By (6.4) and (16), 


$$
\begin{aligned}
& f^{n^{\prime}-n}\left(B_{p_{0}}(90 r / G N)\right) \subset f^{n^{\prime}-n}\left(B_{p_{0}}\left(270 r / \eta\left(p_{0}, G N\right) G N\right)\right) \\
& \quad \subset B_{f^{\prime \prime-n}\left(p_{0}\right)}\left(270 \operatorname{cr} \theta\left(p_{0}, n^{\prime}-n\right) / \eta\left(p_{0}, G N\right)\left(G N-n^{\prime}+n\right)\right),
\end{aligned}
$$

which contradicts (17).

By the same argument, we can see that;

$$
n_{2}-n_{1}>N
$$

From (8), (14), (18) and (8.4), there exists a $\left(p_{0}, N_{0}, f^{n_{1}}\left(p_{0}\right), f^{n_{2}}\left(p_{0}\right), v, a_{1}, a_{2}\right)$ connector $g$ in $\mathscr{U}\left(\varepsilon_{0}, f\right)$. Therefore, $f^{n_{2}-N_{0}}\left(p_{0}\right)$ is a periodic point of $g$ with period $n_{2}-n_{1}$. By the definition of $\mathscr{U}\left(\varepsilon_{0}, f\right)$ (ref. (10.1)), it can be seen that $f^{n_{2}-N_{0}}\left(p_{0}\right)$ must be a saddle of $g$. From the properties of $g$ (ref. (8.3) and (8.2) (iii) (iv)) and (2.3) (ii), we can see that;

$$
E_{f n_{2}-N_{0}\left(p_{0}\right)}^{u}(g)=E_{f^{n_{2}-N_{0}\left(p_{0}\right)}}^{1} .
$$

Therefore, from (2.4),

$$
\left\|\operatorname{Tg}^{n_{2}-n_{1}} \mid E_{f^{n_{2}-N_{0}\left(p_{0}\right)}}^{1}\right\| \geq\left(\lambda^{-1}\right)^{n_{2}-n_{1}} .
$$

By the property of $g$ (ref. (8.2) (iv)), we have;

$$
\left\|T f^{n_{2}-n_{1}} \mid E_{f^{n_{1}\left(p_{0}\right)}}^{1}\right\| \geq\left(\lambda^{-1}\right)^{n_{2}-n_{1}} .
$$

(19) and this (21) imply $\left[n_{1}, n_{2}\right]$ is a $\left(p_{0}, N\right)$-expansive interval in $\left[0, k_{0}\right]$. Recall that $p_{0}=f^{k_{i}}(p), q_{0}=f^{k_{j}}(p)$ and $k_{j}-k_{j}=k_{0}$. So, $\left[k_{i}+n_{1}, k_{i}+n_{2}\right]$ is a $(p, N)$-expansive interval in $[0, m]$. This completes the proof.

\section{$\S 13$. Proof of the Theorem}

In this section, we shall prove our Theorem assuming (11.2) Main lemma whose proof will be given in Section 15. Since we have already finished the essential part, the proof of the Theorem itself is now easy.

Proof. From Lemma 3.1 in [6], we have

$$
\Omega(f)=\Lambda_{0}(f) \cup \Lambda_{1}(f) \cup \Lambda_{2}(f) .
$$

From (2.2), $\Lambda_{0}(f)$ and $\Lambda_{2}(f)$ are finite sets and consequently they are hyperbolic sets. Therefore we have only to show that $\Lambda_{1}(f)$ is a hyperbolic set.

Let $T M \mid \Lambda_{1}(f)=E^{1} \oplus E^{2}$ be the $T f$-invariant splitting given in (2.3) (ii). Put $\Lambda=\Lambda_{1}(f)$ as before. It is enough to show that

(1) there exist $c^{\prime}>0$ and $0<\lambda^{\prime}<1$ such that 
(i) $\left\|T f^{-n} \mid E_{p}^{1}\right\| \leq c^{\prime} \lambda^{\prime n}$,

(ii) $\left\|T f^{n} \mid E_{p}^{2}\right\| \leq c^{\prime} \lambda^{\prime n}, \quad$ for all $n \in Z_{+}$and $p \in \Lambda$.

We shall only prove (i). The same argument as (i) with $f^{-1}$ on $E^{2}$ gives (ii).

Suppose that (i) does not hold, then it follows that

$$
\text { spec. } \operatorname{rad} . f_{*}^{-1} \mid \Sigma^{b}\left(E^{1}\right) \geq 1 \text {. }
$$

From (4.1), we have that

(2) there is a recurrent point $p_{*} \in \Lambda$ such that

$$
\left\|T f^{k} \mid E_{p_{*}}^{1}\right\| \leq 1 \quad \text { for all } k \in Z_{+} .
$$

We fix a positive integer $N \geq N_{4}$. From (12.3), there exists a $\left(p_{*}, N\right)$ expansive interval. Let $I=[m, n]$ be the minimum element of the set of all $\left(p_{*}, N\right)$-expansive intervals, where the order for intervals is given lexicographically, that is; for $I_{1}=\left[m_{1}, n_{1}\right]$ and $I_{2}=\left[m_{2}, n_{2}\right], I_{1} \leq I_{2}$ iff $m_{1}$ $<m_{2}$ or, $m_{1}=m_{2}$ and $n_{1} \leq n_{2}$.

From the definition of the expansive interval, we have

$$
\begin{aligned}
\left\|T f^{n} \mid E_{p_{*}}^{1}\right\| & =\left\|T f^{n-m}\left|E_{f^{m}\left(p_{*}\right.}^{1}\|\cdot\| T f^{m}\right| E_{p_{*}}^{1}\right\| \\
& \geq\left(\lambda^{-1 / 2}\right)^{N} \cdot\left\|T f^{m} \mid E_{p_{*}}^{1}\right\| .
\end{aligned}
$$

From (2), we have

$$
\log \left\|T f^{m} \mid E_{p_{*}}^{1}\right\| \leq(N / 2)(\log \lambda) .
$$

Then, by (12.2), it follows that there exists a $\left(p_{*}, N\right)$-expansive interval in $[0, m]$. This contradicts the fact that $I=[m, n]$ in the minimum among all $\left(p_{*}, N\right)$-expansive intervals. This proves the Theorem.

\section{§14. Taper neighborhoods}

In this section, we shall introduce for each $p \in \Lambda$ a certain class of neighborhoods with special shape which plays an essential role in the proof of the Main lemma in Section 15.

For notational convenience, we put

$$
\alpha=\lambda^{1 / 20}, \quad \beta=\lambda^{-1 / 2} .
$$

(14.1) Definition. Let $r^{\prime}>r>0$. We define a subset of $\boldsymbol{R}^{2}$ by

$$
T\left(r, r^{\prime}\right)=\bigcup_{0 \leq t \leq\left(\log r^{\prime} / r\right) /(\log \beta)} B\left(r \alpha^{t}, r \beta^{t}\right) .
$$


In other words, $(x, y) \in T\left(r, r^{\prime}\right)$ iff there exists $0 \leq t \leq\left(\log r^{\prime} / r\right) /(\log \beta)$ such that $|x| \leq r \alpha^{t},|y| \leq r \beta^{t}$.

By elementary computations, we can see that

$$
s\left(T\left(r, r^{\prime}\right)\right)=(4 / 9) r^{11 / 10}\left(10 r^{/ 9 / 10}-r^{9 / 10}\right),
$$

where $s(\quad)$ denotes the area. Since $r^{\prime}>r>0$, we have

(14.2) Lemma.

$$
s\left(T\left(r, r^{\prime}\right)\right)>4 r^{11 / 10} \cdot r^{\prime / 10}
$$

It is very important that $s\left(T\left(r, r^{\prime}\right)\right)$ has order $11 / 10$ with respect to $r$ (ref. (11) in Section 15).

(14.3) Definition. Let $r_{1} / 2>r^{\prime}>r>0$ and $p \in \Lambda$. We define $T_{p}\left(r, r^{\prime}\right)$ $=\psi_{p}^{-1}\left(T\left(r, r^{\prime}\right)\right)$.

We call such a neighborhood $T_{p}\left(r, r^{\prime}\right)$, taper neighborhood. By drawing the picture, one can see the reason of the name.

Remark that, since $T\left(r, r^{\prime}\right)$ is symmetric with respect to $x$ and $y$-axes in $R^{2}, T_{p}\left(r, r^{\prime}\right)$ is independent of the choice of the basis of $E_{p}^{1} \oplus E_{p}^{2}$ with which $\psi$ is defined.

The iterated images of $T_{p}\left(r, r^{\prime}\right)$ under $f$ are given as follows.

(14.4) LEMMA. Let $r_{9}=\min \left\{r_{1} / 2,\left(\lambda^{-1 / 20}-1\right) / 2 M_{1} c\right\}, r_{9}>r^{\prime}>r>0$ and $p \in \Lambda$. If

$$
\left\|T f^{n} \mid E_{p}^{1}\right\| \leq 1 \quad \text { for all } 0 \leq n \leq\left[\left(\log r^{\prime} / r\right) /(\log \beta)\right]+1,
$$

then

$$
f^{i}\left(B_{p}\left(r \alpha^{t}, r \beta^{t}\right)\right) \subset B_{f^{i}(p)}\left(\theta(p, i) r \alpha^{t-i}, \omega(p, i) r \beta^{t-i}\left(\lambda^{11 / 20}\right)^{i}\right)
$$

for all $0 \leq t \leq\left(\log r^{\prime} / r\right) /(\log \beta)$ and integers $0 \leq i \leq[t+1]$.

Proof. We fix $0 \leq t \leq\left(\log r^{\prime} / r\right) /(\log \beta)$ and prove this lemma by induction on $i=0,1, \cdots,[t+1]$.

When $i=0$, it is trivial. Assuming that the lemma is true for $i-1$, we show that,

$$
\begin{gathered}
f\left(B_{f^{i-1}(p)}\left(\theta(p, i-1) r \alpha^{t-i+1}, \omega(p, i-1) r \beta^{t-i+1}\left(\lambda^{-11 / 20}\right)^{i-1}\right)\right. \\
\subset B_{f^{i}(p)}\left(\theta(p, i) r \alpha^{t-i}, \omega(p, i) r \beta^{t-i}\left(\lambda^{-11 / 20}\right)^{i}\right) .
\end{gathered}
$$

Let $\left(f_{1}, f_{2}\right)=\psi_{f^{i}(p)} \circ f \circ \psi_{f^{i-1}(p)}^{-1}$, and $(x, y) \in \psi_{f^{i-1}(p)}\left(B_{f^{i-1}(p)}(-)\right)$. It is enough to show that 


$$
\begin{aligned}
& \left|f_{1}(x, y)\right| \leq \theta(p, i) r \alpha^{t-i} \\
& \left|f_{2}(x, y)\right| \leq \omega(p, i) r \beta^{t-i}\left(\lambda^{-11 / 20}\right)^{i} .
\end{aligned}
$$

By using the fact that $\theta(p, i-1) \leq 1, \omega(p, i-1) \leq c \lambda^{i-1} \theta(p, i-1)$ and $i \leq[t+1]$ i.e. $t-i+1 \geq 0$, we have

$$
\begin{aligned}
|x|+|y| & \leq \theta(p, i-1) r \alpha^{t-i+1}+\omega(p, i-1) r \beta^{t-i+1}\left(\lambda^{-11 / 20}\right)^{i-1} \\
& \leq r\left(\alpha^{t-i+1}+\beta^{t-i+1} c\left(\lambda^{9 / 20}\right)^{i-1}\right) \\
& \leq r\left(1+c \beta^{t-i+1}\right) \leq r+c r \beta^{t} \\
& \leq r+c r^{\prime} \leq 2 c r^{\prime}
\end{aligned}
$$

So, by the hypothesis on $r^{\prime}$, we have

$$
1+M_{1}(|x|+|y|) \leq \lambda^{-1 / 20} .
$$

By (5.6),

$$
\begin{aligned}
\left|f_{1}(x, y)\right| & \leq|x| \cdot\left\|T f \mid E_{f^{i-1}(p)}^{1}\right\|\left\{1+M_{1}(|x|+|y|)\right\} \\
& \leq \theta(p, i-1) r \alpha^{t-i+1}\left\|T f \mid E_{f^{i-1}(p)}^{1}\right\| \cdot \lambda^{-1 / 20} \\
& =\theta(p, i) r \alpha^{t-i} \\
\left|f_{2}(x, y)\right| & \leq|y| \cdot\left\|T f \mid E_{f^{i-1}(p)}^{2}\right\|\left\{1+M_{1}(|x|+|y|)\right\} \\
& \leq \omega(p, i-1) r \beta^{t-i+1}\left(\lambda^{-11 / 20}\right)^{i-1}\left\|T f \mid E_{f^{i-1}(p)}^{2}\right\| \lambda^{-1 / 20} \\
& =\omega(p, i) r \beta^{t-i}\left(\lambda^{-11 / 20}\right)^{i}
\end{aligned}
$$

This completes the proof.

Let $0 \leq t \leq\left(\log r^{\prime} / r\right) /(\log \beta)$ and $i=[t+1]$. Then, since $-1 \leq t-i$ $\leq 0$, we have $\alpha^{t-i} \leq \alpha^{-1}=\lambda^{-1 / 20} \leq \lambda^{-1}$ and $\beta^{t-i} \leq 1$, and since $\omega(p, i) \leq$ $c \lambda^{i} \theta(p, i), \omega(p, i)\left(\lambda^{-11 / 20}\right)^{i} \leq c \theta(p, i)\left(\lambda^{9 / 20}\right)^{i} \leq c \theta(p, i)$. So, from (14.4), we have

$$
f^{i}\left(B_{p}\left(r \alpha^{t}, r \beta^{t}\right)\right) \subset B_{f^{i}(p)}\left(\theta(p, i) r \lambda^{-1}, \theta(p, i) r c\right) .
$$

Thus, recalling that $\lambda^{-1} \leq 2$ from (2.5), we get;

(14.5) Lemma. Let $r_{9}>r^{\prime}>r>0$ and $p \in \Lambda$. If

$$
\left\|T f^{n} \mid E_{p}^{1}\right\| \leq 1 \quad \text { for all } 0 \leq n \leq\left[\left(\log r^{\prime} / r\right) /(\log \beta)\right]+1,
$$

then for any $z \in T_{p}\left(r, r^{\prime}\right)$, there is an integer $0 \leq i \leq\left[\left(\log r^{\prime} / r\right) /(\log \beta)\right]+1$ such that

$$
f^{i}(z) \in B_{f^{i}(p)}(2 \operatorname{cr} \theta(p, i)) \text {. }
$$

Remark. The assumption $\lambda \geq 1 / 2$ (ref. (2.5)) is only used to simplify the notation. The argument in Section 15 works similarly without it. 


\section{§15. Proof of (11.2) Main lemma}

In this section, we shall give a proof of (11.2) Main lemma. It is rather hard but completes our whole work.

Let $p \in \Lambda, m \in Z_{+}$and $N \in Z_{+}$. Assume that

$$
\log \left\|T f^{m} \mid E_{p}^{1}\right\| \leq\left(N^{10 / 9} / 2\right)(\log \lambda) .
$$

We want to show that, if $N$ is sufficiently large, then we can select a positive integer $m^{\prime}<m$ such that

$$
\left\|T f^{n} \mid E_{f^{m^{\prime}(p)}}^{1}\right\| \leq 1 \quad \text { for all } 0 \leq n \leq m-m^{\prime}
$$

and construct pairwise disjoint intervals in $\left[0, m-m^{\prime}\right]$ with the properties given in the statement of (11.2).

As the first step, we choose a suborbit of the positive orbit of $p \in \Lambda$ which is nearly recurrent and satisfies a good condition for norms of differential of $f$ restricted to $E^{1}$.

Let $0<n_{1} \leq m$ be the largest integer with the property;

$$
\log \left\|T f^{n_{1}} \mid E_{p}^{1}\right\| \geq\left(N^{10 / 9} / 4\right)(\log \lambda)+\left(\log m_{0}\right) .
$$

If we take $N$ large enough to satisfy

$$
N^{10 / 9}>G_{1}^{-1},
$$

then by easy computations, it can be seen that

$$
\begin{aligned}
& \log \left\|T f^{n_{1}} \mid E_{p}^{1}\right\|<\left(N^{10 / 9} / 4\right)(\log \lambda), \\
& \log \left\|T f^{m-n_{1}} \mid E_{f^{n_{1}(p)}}^{1}\right\|<\left(N^{10 / 9} / 8\right)(\log \lambda) .
\end{aligned}
$$

Recalling (11.4), we have

(6) If $q \in \Lambda, n \in Z_{+}$and $\ell>0$ satisfy

$$
\log \left\|T f^{n} \mid E_{q}^{1}\right\| \leq-\ell, \quad \text { then } n \geq\left(\log m_{0}^{-1}\right)^{-1} \cdot \ell .
$$

Then, from (5) and (6),

$$
m-n_{1} \geq G_{1} N^{10 / 9} .
$$

By applying (2.7) to (4) with $\rho=1$, we find $\nu_{1}=\left[N^{10 / 9}\left(\log \lambda^{-1}\right) / 4\left(\log m_{0}^{-1}\right)\right]$ +1 integers $0 \leq k_{1}<k_{2}<\cdots<k_{\nu_{1}} \leq n_{1}$ with the property that;

(8) $\left\|T f^{n} \mid E_{f k_{i(p)}}^{1}\right\| \leq 1 \quad$ for all $0 \leq n \leq n_{1}-k_{i}$ and $1 \leq i \leq \nu_{1}$.

From the definition of $n_{1}$ and (8). 


$$
\left\|T f^{n} \mid E_{f^{k i(p)}}^{1}\right\| \leq 1 \quad \text { for all } 0 \leq n \leq m-k_{i} .
$$

We fix $r>0$ such that

$$
180 c^{4} r<\min \left\{r_{0}, r_{3}, r_{4}, r_{9}\right\},
$$

and consider the taper neighborhoods $T_{f^{k} i(p)}(r / G N, r)$ for each $1 \leq i \leq \nu_{1}$. Let us estimate the sum of the area of these taper neighborhoods. From (14.2) and (5.4) (vi),

$$
\begin{aligned}
\sum_{i=1}^{v_{1}} s\left(T_{f^{k i}(p)}(r / G N, r)\right) & \geq \sum_{i=1}^{v_{1}} c_{1} \cdot s(T(r / G N, r))>\nu_{1} \cdot c_{1} \cdot 4 \cdot(r / G N)^{11 / 10} \cdot r^{9 / 10} \\
& =\left\{\left(\log \lambda^{-1}\right) /\left(\log m_{0}^{-1}\right)\right\} \cdot c_{1} \cdot G^{-11 / 10} \cdot r^{2} \cdot N^{1 / 90}
\end{aligned}
$$

which exceeds the surface area of $M$ by taking $N$ large enough. So, we have

(11) there are at least two points $f^{k_{i}}(p), f^{k_{j}}(p)$ such that;

$$
T_{f k_{i(p)}}(r / G N, r) \cap T_{f^{k} j(p)}(r / G N, r) \neq \phi .
$$

We assume that $k_{j}>k_{i}$, and put;

$$
f^{k_{2}}(p)=p_{0}, f^{k_{j}}(p)=q_{0} \quad \text { and } \quad k_{j}-k_{i}=\tau \text {, so } f^{\tau}\left(p_{0}\right)=q_{0} .
$$

From (7), (8), (9),

$$
\begin{array}{ll}
\left\|T f^{n} \mid E_{p_{0}}^{1}\right\| \leq 1 & \text { for all } 0 \leq n \leq G_{1} N^{10 / 9}+\tau, \\
\left\|T f^{n} \mid E_{q_{0}}^{1}\right\| \leq 1 & \text { for all } 0 \leq n \leq G_{1} N^{10 / 9} .
\end{array}
$$

From (11), we may take a point $z \in T_{p_{0}}(r / G N, r) \cap T_{q_{0}}(r / G N, r)$. We take $N$ large enough to satisfy $(\log \beta)^{-1}(\log G N)+1 \leq G_{1} N^{10 / 9}$, then from (14.5), we have

(14) (i) there is an integer $0 \leq i \leq(\log \beta)(\log G N)+1$ such that $f^{i}(z) \in B_{f^{i}\left(p_{0}\right)}\left(2 c r \theta\left(p_{0}, i\right) / G N\right)$,

(ii) there is an integer $0 \leq j \leq(\log \beta) \cdot(\log G N)+1$ such that $f^{j}(z) \in B_{f^{j}\left(q_{0}\right)}\left(2 \operatorname{cr} \theta\left(q_{0}, j\right) / G N\right)$.

Take large $N$ such that

$$
(\log \beta)^{-1}(\log G N)+1<\left[G_{1} N\right]<[G N]<G_{1} N^{10 / 9} .
$$

We put $\tau_{1}=\left[G_{1} N\right]$, then from (13) and (6.4),

$$
\begin{aligned}
& f^{\tau_{1}-i}\left(B_{f^{i}\left(p_{0}\right)}\left(2 \operatorname{cr} \theta\left(p_{0}, i\right) / G N\right)\right) \subset B_{f^{\tau_{1}\left(p_{0}\right)}}\left(2 c^{2} r \theta\left(p_{0}, \tau_{1}\right) /\left(G N-\tau_{1}+i\right)\right) \\
& \quad \subset B_{f^{\tau_{1}\left(p_{0}\right)}}\left(2 c^{2} r \theta\left(p_{0}, \tau_{1}\right) /\left(G N-\tau_{1}\right)\right) .
\end{aligned}
$$


So, from (14), we have

$$
f^{\tau_{1}}(z) \in B_{f^{\tau_{1}\left(p_{0}\right)}}\left(2 c^{2} r \theta\left(p_{0}, \tau_{1}\right) /\left(G N-\tau_{1}\right)\right) .
$$

Similarly,

$$
f^{\tau_{1}}(z) \in B_{f^{\tau_{1}\left(q_{0}\right)}}\left(2 c^{2} r \theta\left(q_{0}, \tau_{1}\right) /\left(G N-\tau_{1}\right)\right) .
$$

Let $N_{0} \in Z_{+}$be given in (10.1), and take $N$ so large that,

$$
N \geq 8 N_{0}\left(\log m_{0}^{-1}\right) /\left(\log \lambda^{-1}\right)=G_{1}^{-1} N_{0} .
$$

Put $\nu_{0}=\nu_{0}(N)=\left[G_{1} N / 4 N_{0}\right]$ and for each integer $1 \leq k \leq \nu_{0}$, put

$$
\tau_{k}=\tau_{1}+(k-1) 4 N_{0} \text { and } f^{\tau_{k}}\left(p_{0}\right)=p_{k}, f^{\tau_{k}}\left(q_{0}\right)=q_{k} .
$$

Note that, from (12), $f^{\tau}\left(p_{k}\right)=q_{k}$ for all $1 \leq k \leq \nu_{0}$. Also clearly,

$$
\tau_{k}<2\left[G_{1} N\right] \leq 2 G_{1} N \quad \text { for all } 1 \leq k \leq \nu_{0} .
$$

From (16), (17),

$$
B_{p_{1}}\left(2 c^{2} r \theta\left(p_{0}, \tau_{1}\right) /\left(G N-\tau_{1}\right)\right) \cap B_{q_{1}}\left(2 c^{2} r \theta\left(q_{0}, \tau_{1}\right) /\left(G N-\tau_{1}\right)\right) \neq \phi .
$$

By (6.1),

(i ) if $\theta\left(p_{0}, \tau_{1}\right) \geq \theta\left(q_{0}, \tau_{1}\right)$, then $q_{1} \in B_{p_{1}}\left(6 c^{2} r \theta\left(p_{0}, \tau_{1}\right) /\left(G N-\tau_{1}\right)\right)$,

(ii) if $\theta\left(p_{0}, \tau_{1}\right) \leq \theta\left(q_{0}, \tau_{1}\right)$, then $p_{1} \in B_{q_{1}}\left(6 c^{2} r \theta\left(q_{0}, \tau_{1}\right) /\left(G N-\tau_{1}\right)\right)$.

So, from (9), in either case, we have

(20) there exist $p_{*} \in \Lambda$ such that

$$
\begin{aligned}
& \left\|T f^{n} \mid E_{p_{*}}^{1}\right\| \leq 1 \quad \text { for all } 0 \leq n \leq G_{1} N^{10 / 9} \text { and } \\
& p_{1}, q_{1} \in B_{f^{\tau_{1}\left(p_{*}\right)}}\left(6 c^{2} r \theta\left(p_{*}, \tau_{1}\right) /\left(G N-\tau_{1}\right)\right) .
\end{aligned}
$$

In fact, we can take $p_{0}=p_{*}$ (resp. $q_{0}=p_{*}$ ) in case (i) (resp. (ii)). From this (20) and (6.4), we get

$$
p_{k}, q_{k} \in B_{f^{\tau k\left(p_{*}\right)}}\left(6 c^{3} r \theta\left(p_{*}, \tau_{k}\right) /\left(G N-\tau_{k}\right)\right) \quad \text { for all } 1 \leq k \leq \nu_{0} .
$$

Now, since $k_{i}$ in (11) and (12) satisfies the property (i) of the statement of (11.2), we can take $m^{\prime}=k_{i}$ in (11.2). In what follows, we shall construct the disjoint intervals in $\left[0, \tau+\tau_{\nu_{0}}\right]$ with the properties (ii), (iii) in the statement of (11.2). Our strategy is the following. We identify the $Z_{+}$-interval $\left[0, \tau+\tau_{\nu_{0}}\right]$ with the positive orbit of $p_{0}=f^{k_{i}}(p)$, that is $\left\{f^{n}\left(p_{0}\right)\right\}_{0 \leq n \leq \tau+\tau_{\nu_{0}}}=\left\{p_{0} \cdots p_{1} \cdots p \nu_{0} \cdots q_{0} \cdots q_{1} \cdots q \nu_{0}\right\}$. First, we select connectable subintervals inductively by using (21) and (7.4). These intervals 
are not necessarily disjoint. We regard the union of these intervals as a disjoint union of $w_{\nu 0}$ intervals, so $w_{\nu 0}$ is possibly less than $\nu_{0}(N)$. We will prove that these disjoint intervals are exactly the intervals wanted. Concerning the above positive orbit of $p_{0}$, remark that $q_{0}$ can not be contained in $\left\{f^{n}\left(p_{0}\right)\right\}_{0 \leq n \leq \tau_{\nu_{0}}}$, namely, $\tau>\tau_{\nu_{0}}$. In fact, as we will see later, $\tau>N$ and $\tau_{\nu_{0}}<N / 4$.

Before proceedings to the main step of the proof, we shall make some preparations.

From (10), (15), (20) and (10.2), we get

$$
\begin{aligned}
& B_{f^{i}\left(p_{*}\right)}\left(180 c^{4} r \theta\left(p_{*}, i\right) /(G N-i)\right) \\
& \text { are disjoint with each other for } 0 \leq i \leq 2 N .
\end{aligned}
$$

By this, we have the following two lemmas.

(15.1) Lemma. For $\tau_{1} \leq i \leq \tau_{\nu_{0}}$ and $-N_{0} \leq n \leq N_{0}$, any two of

$$
f^{n}\left(B_{f^{i}\left(p_{*}\right)}\left(180 c^{3} r \theta\left(p_{*}, i\right) /(G N-i)\right)\right)
$$

are disjoint when they have different $i+n$.

(15.2) Lemma. Let $x \in M, s \in Z_{+}$and $0 \leq n_{1} \leq n_{2} \leq 2 \tau_{1}$. If

$$
x \in B_{f^{n_{2}\left(p_{*}\right)}}\left(180 c^{3} r \theta\left(p_{*}, n_{2}\right) /\left(G N-n_{2}\right)\right)
$$

and

$$
f^{s}(x) \in B_{f^{n_{1}\left(p_{*}\right)}}\left(180 c^{3} r \theta\left(p_{*}, n_{1}\right) /\left(G N-n_{1}\right)\right)
$$

then $s>N$.

The proofs of (15.1) and (15.2) are straightforward by using (6.4), (18) and (22). (15.1) will guarantee the disjointness of the supports of the perturbations when we apply the closing lemma on several places simultaneously, and (15.2) guarantees the length of the suborbit with such recurrence.

To complete the proof of the Main lemma, we first fix the following notation.

For a set of pairs of integers $\left\{s_{k}<t_{k}\right\}_{1 \leq k \leq \nu_{0}}$ in $\left[\tau_{1}, \tau+\tau_{\nu_{0}}\right]$, we put $J_{k}$ $=\bigcup_{j=1}^{k}\left[s_{j}, t_{j}\right]$ and $J_{0}=\phi$, where $\left[s_{j}, t_{j}\right]$ denotes the $Z_{+}$-interval. Regard $J_{k}$ as a disjoint union of $Z_{+}$-intervals and denote it by $J_{k}=\bigcup_{j=1}^{w_{k}} I_{j}^{k}$ and $I_{j}^{k}=\left[u_{j}^{k}, v_{j}^{k}\right]$, where $I_{1}^{k}<I_{2}^{k}<\cdots<I_{w_{k}}^{k}$. Note that $w_{k} \leq k$. Next we put $p_{*}^{k}=f^{\tau}\left(p_{*}\right)$ for $1 \leq k \leq \nu_{0}$.

Then we give; 
(15.3) Lemma. There exists a set of pairs of integers $\left\{s_{k}<t_{k}\right\}_{1 \leq k \leq \nu_{0}}$ in $\left[\tau_{1}, \tau+\tau_{\nu_{0}}\right]$ with the following properties for all $1 \leq k \leq \nu_{0}$;

(i) There exist $v_{k} \in T_{p_{*}^{k}} M$ and positive numbers $a_{1}^{k}, a_{2}^{k}$ such that $\left(f^{s_{k}}\left(p_{0}\right), f^{t_{k}}\left(p_{0}\right), v_{k}, a_{1}^{k}, a_{2}^{k}\right)$ is $\left(p_{*}^{k}, 180 c^{3} r \theta\left(p_{*}, \tau_{k}\right) /\left(G N-\tau_{k}\right)\right)$-connectable, namely;

(ia ) $\left|v_{k}\right|+a_{1}^{k}+a_{2}^{k} \leq 180 c^{3} r \theta\left(p_{*}, \tau_{k}\right) /\left(G N-\tau_{k}\right)$

(ib ) $(2 \sqrt{2})^{-1} \leq a_{1}^{k} / a_{2}^{k} \leq 2 \sqrt{2}$

(ic ) $f^{s_{k}}\left(p_{0}\right), f^{t_{k}}\left(p_{0}\right) \in b_{p_{*}^{k}}\left(v_{k}, a_{1}^{k}, a_{2}^{k}\right)\left(V_{p_{*}^{k}}(\sqrt{3 / 4})\right)$

(id ) $b_{p_{*}^{k}}\left(v_{k}, a_{1}^{k}, a_{2}^{k}\right)\left(V_{p_{*}^{k}}(1)\right) \subset B_{p_{*}^{k}}\left(180 c^{3} r \theta\left(p_{*}, \tau_{k}\right) /\left(G N-\tau_{k}\right)\right)$.

(ii ) $s_{k}, t_{k} \notin J_{k-1}$.

(iii) If $n \in\left[\tau_{1}, \tau+\tau_{\nu_{0}}\right]-J_{k-1}$ and $n \neq s_{k}$, $t_{k}$, then $f^{n}\left(p_{0}\right) \notin b_{p_{*}^{k}}\left(v_{k}, a_{1}^{k}, a_{2}^{k}\right)\left(V_{p_{*}^{k}}(1)\right)$.

(iv) $t_{k} \leq \tau+\tau_{k}$.

( v ) $s_{k}>4\left[G_{1} N\right]=4 \tau_{1}$.

(vi ) length $\left(I_{j}^{k}\right)=v_{j}^{k}-u_{j}^{k}>N \quad$ for all $1 \leq j \leq w_{k}$.

(vii) $\sum_{k=1}^{w_{k}}$ length $\left(I_{j}^{k}\right)>k N$.

(viii) $\left\|T f^{v_{j}^{k}-u_{j}^{k}} \mid E_{f u_{j}^{1}\left(p_{0}\right)}^{k}\right\| \geq\left(\lambda^{-1}\right)^{v_{j}^{k}-u_{j}^{k}} \quad$ for all $1 \leq j \leq w_{k}$.

If this (15.3) is verified and such integers $s_{k}, t_{k}$ exist for all $1 \leq k \leq \nu_{0}$, then the Main lemma will be established, because from (vii) and (viii), $\left\{I_{j}^{\nu_{0}}\right\}_{1 \leq j \leq w_{\nu_{0}}}$ are exactly the intervals we want.

Proof of (15.3). We shall prove (15.3) by induction on $k=1, \cdots, \nu_{0}$.

First, let us select $s_{1}, t_{1} \in\left[\tau_{1}, \tau+\tau_{\nu_{0}}\right]$. From (21), $p_{1}=f^{\tau_{1}}\left(p_{0}\right)$ and $q_{1}=$ $f^{\tau_{1}}\left(q_{0}\right)=f^{\tau+\tau_{1}}\left(p_{0}\right)$ are contained in $B_{p_{*}^{1}}\left(6 c^{3} r \theta\left(p_{*}, \tau_{1}\right) /\left(G N-\tau_{1}\right)\right)$. So, applying (7.4) with $\left\{f^{n}\left(p_{0}\right): n \in\left[\tau_{1}, \tau+\tau_{\nu_{0}}\right]\right\}$ as a finite set, we have integers $s_{1}, t_{1}$ $\left(s_{1}<t_{1}\right)$ in $\left[\tau_{1}, \tau+\tau_{\nu_{0}}\right], v_{1} \in T_{p_{*}^{1}} M$ and positive numbers $a_{1}^{1}, a_{2}^{1}$ satisfying (i) and (iii) in the statement of (15.3). For $k=1$, (ii) is not necessary. So, let us prove that $s_{1}$ and $t_{1}$ satisfy (iv) $\sim$ (viii).

From (ic) and (id),

$$
f^{s_{1}}\left(p_{0}\right), f^{\iota_{1}}\left(p_{0}\right) \in B_{p_{*}^{1}}\left(180 c^{3} r \theta\left(p_{*}, \tau_{1}\right) /\left(G N-\tau_{1}\right)\right) .
$$

Applying (15.2) with $x=f^{s_{1}}\left(p_{0}\right), s=t_{1}-s_{1}$ and $n_{1}=n_{2}=\tau_{1}$, we get;

$$
t_{1}-s_{1}>N \text {. }
$$

So, (vi) and (vii) are satisfied.

Let us prove (iv), i.e. $t_{1} \leq \tau+\tau_{1}$. Suppose $t_{1}>\tau+\tau_{1}$. As we observed above,

$$
f^{t_{1}}\left(p_{0}\right) \in B_{p_{*}^{1}}\left(180 c^{3} r \theta\left(p_{*}, \tau_{1}\right) /\left(G N-\tau_{1}\right)\right)
$$


On the other hand, from (21) and (6.4), we have

$$
f^{t_{1}}\left(p_{0}\right)=f^{t_{1}-\tau-\tau_{1}}\left(q_{1}\right) \in B_{f^{t_{1}-\tau\left(p_{*}\right)}}\left(6 c^{4} r \theta\left(p_{*}, t_{1}-\tau\right) /\left(G N-t_{1}+\tau\right)\right),
$$

which contradicts (22), because from (19),

$$
t_{1}-\tau<\left(\tau+\tau_{\nu_{0}}\right)-\tau=\tau_{\nu_{0}}<2 G_{1} N \leq N / 4 .
$$

Next, let us prove (viii). From (i), $\left(f^{s_{1}}\left(p_{0}\right), f^{t_{1}}\left(p_{0}\right), v_{1}, a_{1}^{1}, a_{2}^{1}\right)$ is $\left(p_{*}^{1}, 180 c^{3} r \theta\left(p_{*}, \tau_{1}\right) /\left(G N-\tau_{1}\right)\right)$-connectable. By (10), (10.1), (15.1) and (8.4), there exists a $\left(p_{*}^{1}, N_{0}, f^{s_{1}}\left(p_{0}\right), f^{t_{1}}\left(p_{0}\right), v_{1}, a_{1}^{1}, a_{2}^{1}\right)$-connector $g$ in $\mathscr{U}\left(\varepsilon_{0}, f\right)$. From the definition of the connector $g, f^{t_{1}-N_{0}}\left(p_{0}\right)$ is a periodic point of $g$. Moreover, its period is $t_{1}-s_{1}$ and from (10) and (3.1), this periodic point must be a saddle of $g$. From (8.3), (8.2) (iii) (iv) and (2.3) (ii), we can see that

$$
E_{f^{t_{1}-N_{0}\left(p_{0}\right)}}^{u}(g)=E_{f t_{1}-N_{0}\left(p_{0}\right)}^{1}
$$

Therefore, from (2.4),

$$
\left\|\operatorname{Tg}^{t_{1}-s_{1}} \mid E_{f t_{1}-N_{0}\left(p_{0}\right)}^{1}\right\| \geq\left(\lambda^{-1}\right)^{t_{1}-s_{1}} .
$$

From (8.2) (iv), we get,

$$
\left\|T f^{t_{1}-s_{1}} \mid E_{f^{s_{1}\left(p_{0}\right)}}^{1}\right\| \geq\left(\lambda^{-1}\right)^{t_{1}-s_{1}}
$$

which implies (viii).

Finally, we shall prove (v). Suppose $s_{1} \leq 4 \tau_{1}=4\left[G_{1} N\right]$. From (vi) and (viii),

$$
\begin{aligned}
\left\|T f^{t_{1}} \mid E_{p_{0}}^{1}\right\| & =\left\|T f^{t_{1}-s_{1}}\left|E_{f^{s_{1}\left(p_{0}\right)}}^{1}\|\cdot\| T f^{s_{1}}\right| E_{p_{0}}^{1}\right\| \\
& \geq\left(\lambda^{-1}\right)^{t_{1}-s_{1}} \cdot m_{0}^{s_{1}}>\left(\lambda^{-1}\right)^{N} \cdot m_{0}^{s_{1}} .
\end{aligned}
$$

Namely, we have,

$$
\log \left\|T f^{t_{1}} \mid E_{p_{0}}^{1}\right\|>N\left(\log \lambda^{-1}\right)-4 G_{1} N\left(\log m_{0}^{-1}\right)=(N / 2)\left(\log \lambda^{-1}\right),
$$

which contradicts (13), because from (15) and (19),

$$
t_{1} \leq \tau+\tau_{1}<\tau+G_{1} N^{10 / 9},
$$

Next, assuming that (15.3) is valid for all $1 \leq i \leq k-1$, we prove that there exist integers $s_{k}$ and $t_{k}$ in $\left[\tau_{1}, \tau+\tau_{\nu_{0}}\right]$ with the properties (i) $\sim$ (viii).

Let $Q=\left\{f^{n}\left(p_{0}\right): n \in\left[\tau_{1}, \tau+\tau_{\nu_{0}}\right]-J_{k-1}\right\}$. Since $s_{i}>4 \tau_{1}$ and $t_{i} \leq \tau+\tau_{i}$ for all $1 \leq i \leq k-1, p_{k}=f^{\tau k}\left(p_{0}\right)$ and $q_{k}=f^{\tau_{k}+\tau}\left(p_{0}\right)$ are contained in $Q$. Moreover, from (21), 


$$
p_{k}, q_{k} \in B_{p_{*}^{k}}\left(6 c^{3} r \theta\left(p_{*}, \tau_{k}\right) /\left(G N-\tau_{k}\right)\right) .
$$

So, applying (7.4) with $Q$ as a finite set, we get $v_{k} \in T_{p_{*}^{k}} M$, positive numbers $a_{1}^{k}, a_{2}^{k}$ and integers $s_{k}, t_{k} \in\left[\tau_{1}, \tau+\tau_{\nu_{0}}\right]-J_{k-1}\left(s_{k}<t_{k}\right)$ satisfying (i), (ii) and (iii).

By the same argument as in case of $k=1$, we can see that $t_{k} \leq \tau$ $+\tau_{k}$, i.e. (iv). Let us prove that $s_{k}$ and $t_{k}$ satisfy (v) $\sim$ (viii).

Consider $\left\{I_{j}^{k-1}\right\}_{1 \leq j \leq w_{k-1}}$. Since (ii) holds for all $1 \leq i \leq k$, we have;

(24) Each $I_{j}^{k-1}$ satisfies $I_{j}^{k-1} \subset\left[s_{k}, t_{k}\right]$ or $I_{j}^{k-1} \cap\left[s_{k}, t_{k}\right]=\phi$.

(25) Each $I_{j}^{k-1}$ coincides with some of $\left\{\left[s_{i}, t_{i}\right]\right\}_{1 \leq i \leq k-1}$.

Let $L_{1}<L_{2}<\cdots<L_{w}$ be intervals in $\left\{I_{j}^{k-1}\right\}_{1 \leq j \leq w_{k-1}}$ satisfying $I_{j}^{k-1} \subset$ $\left[s_{k}, t_{k}\right]$. If there are no such intervals, that is; $I_{j}^{k-1} \cap\left[s_{k}, t_{k}\right]=\phi$ for all $1 \leq j \leq w_{k-1}$, then by the same argument as in case of $k=1$, it can be seen that $\left\{s_{i}, t_{i}\right\}_{1 \leqslant i \leqslant k}$ satisfy the properties (i) $\sim$ (viii). So, we assume that $w \geq 1$.

From (25), for any $1 \leq n \leq w$, we can put $L_{n}=\left[s_{i_{n}}, t_{i_{n}}\right]$ with some $1 \leq i_{n} \leq k-1$. From the definition of $\left\{L_{n}\right\}_{1 \leq n \leq w}$, we have;

$$
s_{k}<s_{i_{1}} .
$$

Since (ic) and (id) hold for all $1 \leq i \leq k$,

$$
f^{s_{i_{1}}}\left(p_{0}\right) \in B_{p_{*}^{i_{1}}}\left(180 c^{3} r \theta\left(p_{*}, \tau_{i_{1}}\right) /\left(G N-\tau_{i_{1}}\right)\right)
$$

and

$$
f^{s_{k}}\left(p_{0}\right) \in B_{p_{*}^{k}}\left(180 c^{3} r \theta\left(p_{*}, \tau_{k}\right) /\left(G N-\tau_{k}\right)\right) .
$$

So, applying (15.2) with $x=f^{s_{k}}\left(p_{0}\right), s=s_{i_{1}}-s_{k}, n_{1}=\tau_{i_{1}}$ and $n_{2}=\tau_{k}$, we get

$$
s_{i_{1}}-s_{k}>N \text {. }
$$

From this fact and the hypothesis of induction, one can easily see that $\left\{s_{i}, t_{i}\right\}_{1 \leq i \leq k}$ satisfy (vi) and (vii).

If (viii) is proved, by the same argument as in case of $k=1$ using (vi) and (viii), we can verify (v). Thus, we shall finish the proof of (15.3) (and so the Main lemma) by proving (viii).

Since $1 \leq i_{n} \leq k-1$, from (i), $\left(f^{s_{i_{n}}}\left(p_{0}\right), f^{t_{i_{n}}}\left(p_{0}\right), v_{i_{n}}, a_{1}^{i_{n}}, a_{2}^{i_{n}}\right)$ is $\left(p_{*}^{i_{n}}, 180 c^{3} r \theta\left(p_{*}, \tau_{i_{n}}\right) /\left(G N-\tau_{i_{n}}\right)\right)$-connectable. By (id), (10.1), (15.1) and (8.4),

(26) there exists a $\left(p_{*}^{i_{n}}, N_{0}, f^{t_{i n}}\left(p_{0}\right), f^{s_{i}}\left(p_{0}\right), v_{i_{n}}, a_{1}^{i_{n}}, a_{2}^{i_{n}}\right)$-connector $g_{n}$ in $\mathscr{U}\left(\varepsilon_{0}, f\right)$. 
And since (i) holds for $k,\left(f^{s_{k}}\left(p_{0}\right), f^{t_{k}}\left(p_{0}\right), v_{k}, a_{1}^{k}, a_{2}^{k}\right)$ is $\left(p_{*}^{k}, 180 c^{3} r \theta\left(p_{*}, \tau_{k}\right) /\right.$ $\left.\left(G N-\tau_{k}\right)\right)$-connectable, and similarly;

(27) there exists a $\left(p_{*}^{k}, N_{0}, f^{s_{k}}\left(p_{0}\right), f^{t_{k}}\left(p_{0}\right), v_{k}, a_{1}^{k}, a_{2}^{k}\right)$-connector $g_{0}$ in $\mathscr{U}\left(\varepsilon_{0}, f\right)$.

It is important to remark that $f^{t_{i}}\left(p_{0}\right)$ appears before $f^{s_{n}}\left(p_{0}\right)$ in (26) and $f^{s_{k}}\left(p_{0}\right)$ appears before $f^{t_{k}}\left(p_{0}\right)$ in (27). From (8.3) and (8.2), $g_{n}$ and $g_{0}$ have the property that

$$
\begin{aligned}
& g_{n}^{2 N_{0}}\left(f^{s_{i_{n}}-N_{0}}\left(p_{0}\right)\right)=f^{t_{i_{n}}+N_{0}}\left(p_{0}\right) \\
& g_{0}^{2 N_{0}}\left(f^{t_{k}-N_{0}}\left(p_{0}\right)\right)=f^{s_{k}+N_{0}}\left(p_{0}\right) .
\end{aligned}
$$

It means that $g_{n}$ cuts off the suborbit $\left\{f^{n}\left(p_{0}\right): s_{i_{n}} \leq n \leq t_{i_{n}}\right\}$. It seems that $g_{0}$ closes up the suborbit $\left\{f^{n}\left(p_{0}\right): s_{k} \leq n \leq t_{k}\right\}$. But, $f^{t_{k}-N_{0}}\left(p_{0}\right)$ is not necessarily a periodic point of $g_{0}$, because from (iii), the points $\left\{f^{n}\left(p_{0}\right): n \in\right.$ $\left.\bigcup_{j=1}^{w} L_{j}\right\}$ may be contained in the support of $g_{0} f^{-1}$. We construct a periodic point by cutting off these intervals $\left\{L_{n}\right\}_{1 \leq n \leq w}$ by $g_{n}$.

We put,

$$
h_{0}=g_{0} f^{-1} \quad \text { and } \quad h_{n}=g_{n} f^{-1} \quad \text { for } 1 \leq n \leq w,
$$

and

$$
g=h_{w} \circ h_{w-1} \circ \cdots \circ h_{1} \circ h_{0} \circ f .
$$

From (26), (27), (id), (8.2) (ii) and (15.1),

$$
\left\{\operatorname{supp} h_{n}\right\} \quad \text { are disjoint for } 0 \leq n \leq w \text {. }
$$

Since $g_{n} \in \mathscr{U}\left(\varepsilon_{0}, f\right)$ for all $0 \leq n \leq w$, from (2.8), we have

$$
g \in \mathscr{U}\left(\varepsilon_{0}, f\right) .
$$

Let us consider the positive orbit of $f^{t_{k}-N_{0}}\left(p_{0}\right)$ for $g$. We put

$$
\begin{aligned}
& g^{N_{0}}\left(f^{t_{k}-N_{0}}\left(p_{0}\right)\right)=x_{0}, \\
& g^{N_{0}}\left(f^{s_{i_{n}}-N_{0}}\left(p_{0}\right)\right)=x_{n} \quad \text { for } 1 \leq n \leq w .
\end{aligned}
$$

Then, from (26), (27), (30), (ic), (id) and (iii), we get;

$$
\begin{aligned}
& g^{s_{1}-s_{k}}\left(x_{0}\right)=x_{1}, \\
& g^{s_{i_{n}}-t_{i_{n-1}}}\left(x_{n-1}\right)=x_{n} \quad \text { for all } 2 \leq n \leq w, \\
& g^{t_{k}-t_{w}}\left(x_{w}\right)=x_{0} .
\end{aligned}
$$

Thus, $x_{0}$ is a periodic point of $g$ with period

$$
\left(t_{k}-s_{k}\right)-\sum_{n=1}^{w}\left(t_{i_{n}}-s_{i_{n}}\right) .
$$


Since $g \in \mathscr{U}(\varepsilon, f)$, from (10.1) and (3.1), $x_{0}$ must be a saddle of $g$.

Now, it holds that $E_{x_{0}}^{u}(g)=E_{x_{0}}^{1}$ and $E_{x_{0}}^{s}(g)=E_{x_{0}}^{2}$. In fact, as $g$ preserves $E^{1}$ and $E^{2}$ on the orbit of the saddle $x_{0}, E_{x_{0}}^{1}$ and $E_{x_{0}}^{2}$ must be either $E_{x_{0}}^{u}(g)$ or $E_{x_{0}}^{s}(g)$. From (33) and the properties of the connector (8.3) and (8.2) (iii), (iv), we have;

(34) for $j=1,2$,

$$
\begin{aligned}
& \left\|T g^{s_{i_{1}}-s_{k}}\left|E_{x_{0}}^{j}\|=\| T f^{s_{i_{1}}-s_{k}}\right| E_{f s_{k}\left(p_{0}\right)}^{j}\right\| \\
& \left\|T g^{s_{i_{n}}-t_{i_{n-1}}}\left|E_{x_{n-1}}^{j}\|=\| T f^{s_{i_{n}}-t_{i_{n-1}}}\right| E_{f i_{n-1}\left(p_{0}\right)}^{j}\right\|
\end{aligned}
$$

for all $2 \leq n \leq w$, and

$$
\left\|T g^{t_{k}-t_{i_{w}}}\left|E_{x_{w}}^{j}\|=\| T f^{t_{k}-t_{i_{w}}}\right| E_{f i_{w}\left(p_{0}\right)}^{j}\right\| .
$$

From the construction of $s_{i}, t_{i}$, it is clear that

$$
s_{i_{n}}-t_{i_{n}} \geq 4 N_{0} \quad \text { for all } 2 \leq n \leq w,
$$

and

$$
t_{k}-t_{i_{w}} \geq 4 N_{0}
$$

And as we observed above, $s_{i_{1}}-s_{k}>N$. If we take $N$ large so that $c \lambda^{N}$ $<1$, then from (2.3) (ii), (34) and the hypothesis of $N_{0}$ in (10.1) (i.e. $c \lambda^{N_{0}}$ $\leq 1$ ), we have,

$$
\left\|\operatorname{Tg}^{\pi\left(x_{0}\right)}\left|E_{x_{0}}^{2}\|\cdot\| \operatorname{Tg}^{\pi\left(x_{0}\right)}\right| E_{x_{0}}^{1}\right\|^{-1}<1
$$

where $\pi\left(x_{0}\right)$ denotes the period. So, from (2.4), we have that

$$
E_{x_{0}}^{u}(g)=E_{x_{0}}^{1}, \quad E_{x_{0}}^{s}(g)=E_{x_{0}}^{2},
$$

and moreover,

$$
\left\|\operatorname{Tg}^{\pi\left(x_{0}\right)} \mid E_{x_{0}}^{1}\right\| \geq\left(\lambda^{-1}\right)^{\pi\left(x_{0}\right)} .
$$

From the hypothesis of induction,

$$
\left\|T f^{t_{i_{n}}-s_{i_{n}}} \mid E_{f}^{1} s_{i_{n}\left(p_{0}\right)}\right\| \geq\left(\lambda^{-1}\right)^{t_{i_{n}-s_{i_{n}}}}
$$

for all $1 \leq n \leq w$. Therefore, from (34), (35) and the fact that $\operatorname{dim} E^{1}=$ 1 , we get (viii) for $k$.

This completes the proof of (15.3) and so (11.2) Main lemma. 


\section{REFERENCES}

[1] J. Franks, Necessary conditions for stability of diffeomorphisms, Trans. Amer. Math. Soc., 158 (1971), 301-308.

[2] M. Hirsch, J. Palis, C. Pugh and M. Shub, Neighborhoods of hyperbolic sets, Invent. Math., 9 (1970), 121-134.

[3] M. Hirsch and C. Pugh, Stable manifolds and hyperbolic sets, Proc. of Sympo. Pure Math. (Global Analysis) XIV, Amer. Math. Soc., (1970), 133-163.

[ 4 ] M. Hirsch, C. Pugh and M. Shub, Invariant Manifolds, Lec. Notes in Math. 583, Springer.

[5] R. Mañé, Expansive diffeomorphisms. Dynamical Systems, Warwick, 1974. Lec. Notes in Math. 468, Springer.

[ 6 ] - Contributions to the stability conjecture, Topology, 17 (1978), 383-396.

[ 7 ] J. Mather, Characterization of Anosov diffeomorphisms, Indag. Math., 30 (1968), 479-483.

[8] J. Palis, A note on $\Omega$-stability, Proc. of Sympo. Pure Math. (Global Analysis), XIV, Amer. Math. Soc., 221-222.

[9] J. Palis and S. Smale, Structural stability theorems, Proc. of Sympo. Pure Math. (Global Analysis), XIV, Amer. Math. Soc., (1970), 223-231.

[10] V. A. Pliss, The location of separatrices of periodic saddle-point motion of systems of second-order differential equations, Differential Equations, 7 (1971), 906-927.

[11] — A hypothesis due to Smale, Differential Equations, 8 (1972), 203-214.

[12] C. Pugh and C. Robinson, The $C^{1}$ Closing Lemma, Including Hamiltonians, Preprint.

[13] J. Robbin, A structural stability theorem. Ann. of Math., 94 (1971), 447-493.

[14] C. Robinson, $C^{\text {r }}$ structural stability implies Kupka-Smale, Dynamical systemsSalvador, M. Peixoto (Editor), Academic Press, 1973.

[15] - Structural stability of $C^{1}$ diffeomorphisms, J. Differential Equations, 22 (1976), 28-73.

[16] - Stability, measure zero, and dimension two implies hyperbolicity, Preprint.

[17] S. Smale, Differentiable dynamical systems, Bull. Amer. Math. Soc., 73 (1967), $747-817$.

[18] - The $\Omega$-stability theorem, Proc. of Sympo. Pure Math. (Global Analysis), 14, Amer. Math. Soc., (1970), 289-297.

Department of Mathematics

Hokkaido University

Sapporo, Japan 